\title{
III. X-RAY MINERALOGY DATA, AUSTRAL-ANTARCTIC REGION, LEG 28, DEEP SEA DRILLING PROJECT ${ }^{1}$
}

\author{
H. E. Cook, I. Zemmels, and J. C. Matti, University of California, Riverside, California
}

\section{METHODS}

Semiquantitative determinations of the mineral composition of bulk samples, $2-20 \mu \mathrm{m}$, and $<2 \mu \mathrm{m}$ fractions were performed according to the methods described in the appendix of this volume.

The X-ray mineralogy results of this study are summarized in Tables 1 through 11. The mineralogy data are presented in Tables 12 through 26 . Sediment ages, lithologic units, and nomenclature of the sediment types in Tables 1 through 11 are from the DSDP Leg 28 Hole Summaries and from a subsequent update supplied by Dr. Ansis Kaneps, DSDP. The stratigraphic position of samples submitted for X-ray diffraction analysis from Leg 28 are listed in Tables 1 through 11. The sample depth (in $\mathrm{m}$ ) below the sea floor in Tables 1 through 11 identifies the samples as they are reported in Tables 12 through 26.

The method of sample preparation, in brief, is as follows: Bulk samples are washed to remove seawater salts and are ground to less than $10 \mu \mathrm{m}$ under butanol. A portion of the sediment is decalcified in a sodiumacetate-buffered, acetic-acid solution $(\mathrm{pH} 4.5)$. The residue is fractionated into $2-20 \mu \mathrm{m}$ and $<2 \mu \mathrm{m}$ samples by wet sieving and centrifugation. The $2-20 \mu \mathrm{m}$ samples are ground to less than $10 \mu \mathrm{m}$. These three preparations are treated with trihexylamine acetate to expand the smectities. All samples are X-rayed as random powders.

The amorphous content (largely consisting of biogenous silica, volcanic glass, allophane, and organic matter) is computed from the diffuse scatter of a sample. This method assumes that the diffuse scatter in excess of the diffuse scatter from the crystalline materials is a measure of the amorphous content. The diffuse scatter of the crystalline minerals is determined from the mineral calibration standards. Ideally the amorphous content varies between 0 and $100 \%$, but, in cases where the minerals in the sample have a higher degree of crystallinity than the calibration standards, negative values can result. The negative values are reported as blanks; these samples can be assumed to contain little or no amorphous material.

The crystalline minerals are quantified by the method of mutual ratios using peak heights and concentration factors derived from ratioing the diagnostic peaks of minerals with the major peak of quartz. Unquantifiable minerals, i.e., unidentified minerals and minerals for which standards are not available, are tentatively quantified using a hypothetical concentration factor of 3.0 which is applied to the major peak of the mineral. The concentrations of the quantifiable minerals is summed

\footnotetext{
'Institute of Geophysics and Planetary Physics, University of California, Riverside, California, Contribution No. 74-10.
}

to $100 \%$. The amorphous content and the unquantifiable minerals are not included in the total. The unquantifiable minerals are reported on a qualitative scale as trace (less than 5\%), present (5-25\%), abundant (25$65 \%$ ), and major (greater than $65 \%$ ).

The precision of the mineral determination is approximately \pm 1 weight percent of the amount present. Because of differences in the crystallinity between the mineral calibration standards and the minerals in the samples, the accuracy of the reported concentrations is often less than the precision of the method allows. In terms of the reported concentration, smectites may vary $\pm 50 \%$; micas, chlorites, cristobalite, tridymite, goethite may vary $\pm 20 \%$; kaolinite, amphibole, augite, the feldspars, the zeolites, palygorskite, sepiolite, apatite may vary $\pm 10 \%$; the minerals which have stable crystal lattices and are not members of solid-solution series or typically have limited crystal-lattice substitution in the sedimentary environment such as quartz, lowmagnesium calcite, aragonite, dolomite, rhodochrosite, siderite, gibbsite, talc, barite, anatase, gypsum, anhydrite, halite, pyrite, hematite, magnetite will vary less than $\pm 5 \%$.

The user of the X-ray mineralogy data should bear in mind that (1) the reported values are not absolute concentrations and some adjustment has to be made for the amorphous content and the unquantifiable minerals, (2) in a homogeneous system of minerals, the mineral concentration trends are reliable because of the precision, but when comparing mineral concentrations between different geographic regions or lithologic units additional information regarding the crystallinity of the minerals is required, (3) the representativeness of the samples selected for X-ray diffraction analysis is the responsibility of the shipboard scientists, and any questions pertaining to this aspect should be directed to them.

\section{DRILLING MUD USAGE}

Drilling mud, containing montmorillonite and barite, was used as follows:

Mud was used at Site 270 between Cores 39 and 40; at Site 272 between Cores 19 and 20, after Core 36, after Core 41, and after Core 43; in Hole 273A after Core 10; and at Site 274 after Core 43 and after Core 44 . Most samples submitted for X-ray diffraction analysis do not occur close to intervals in which drilling mud was used. Barite does not occur in samples potentially contaminated by drilling mud and montmorillonite abundances are not inordinate in any of these samples.

\section{ACKNOWLEDGMENTS}

The writers wish to acknowledge the excellent work of Nicki D. Coursey in the interpretation of X-ray diffraction data, of Paul D. Johnson in X-ray data acquisition and data processing, and of Tom W. Halverson, Jr. in sample preparation. 
TABLE 2

Summary of X-Ray Mineralogy Samples, Sample Depths, Lithology, Age, and X-Ray Diffraction Results, Site 265

\begin{tabular}{|c|c|c|c|c|c|c|c|c|c|c|c|c|}
\hline \multirow{2}{*}{$\begin{array}{c}\text { Sample } \\
\text { (Interval in cm) }\end{array}$} & \multirow{2}{*}{$\begin{array}{c}\text { Sample } \\
\text { Depth Below } \\
\text { Sea Floor (m) }\end{array}$} & \multirow[b]{2}{*}{ Lithology } & \multirow[b]{2}{*}{ Age } & \multicolumn{3}{|c|}{$\begin{array}{c}\text { Bulk Sample } \\
\text { Major Constituent }\end{array}$} & \multicolumn{3}{|c|}{ 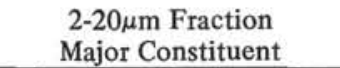 } & \multicolumn{3}{|c|}{$\begin{array}{l}<2 \mu \mathrm{m} \text { Fraction } \\
\text { Major Constituent }\end{array}$} \\
\hline & & & & 1 & 2 & 3 & 1 & 2 & 3 & 1 & 2 & 3 \\
\hline $2-6,100-102$ & 27.0 & $\begin{array}{c}\text { Unit } 1 \\
\text { Clay-bearing }\end{array}$ & Pleistocene & Calc. & & & \multicolumn{3}{|c|}{ Insufficient residue } & \multicolumn{3}{|c|}{ Insufficient residue } \\
\hline $2-6,122-124$ & 27.0 & diatom ooze & & Plag. & Mont. & $\mathrm{K}-\mathrm{Fe}$. & Plag. & Augi. & K-Fe. & Mont. & Plag. & $\mathrm{K}-\mathrm{Fe}$. \\
\hline $\begin{array}{l}5-5,130-132 \\
7-4,120-122\end{array}$ & $\begin{array}{l}111.3 \\
166.7\end{array}$ & $\begin{array}{c}\text { Unit 2 } \\
\text { Micarb-bearing diatom } \\
\text { ooze, with forams } \\
\text { and nanno }\end{array}$ & Pleistocene & $\begin{array}{l}\text { Mont. } \\
\text { Calc. }\end{array}$ & Plag. & $\mathrm{K}-\mathrm{Fe}$. & $\begin{array}{l}\text { Plag. } \\
\text { Ins }\end{array}$ & $\begin{array}{l}\text { Augi. } \\
\text { ficient } r\end{array}$ & $\begin{array}{l}\text { K-Fe. } \\
\text { due }\end{array}$ & $\begin{array}{l}\text { Mont. } \\
\text { Inst }\end{array}$ & $\begin{array}{l}\text { Plag. } \\
\text { ficient re }\end{array}$ & $\begin{array}{l}\text { K-Fe. } \\
\text { due }\end{array}$ \\
\hline $14-6,130-132$ & 369.3 & $\begin{array}{c}\text { Unit } 3 \\
\text { Clay-bearing } \\
\text { diatom ooze }\end{array}$ & Pliocene & Mica & Plag. & Quar. & $\mathrm{K}-\mathrm{Fe}$. & Plag. & Quar. & Mont. & $\mathrm{K}-\mathrm{Fe}$. & Mica \\
\hline $15-4,78-80$ & 403.8 & $\begin{array}{l}\text { Unit } 4 \\
\text { Clay and diatom- } \\
\text { bearing nanno ooze } \\
\text { to chalk }\end{array}$ & $\begin{array}{l}\text { Middle } \\
\text { Miocene }\end{array}$ & Calc. & & & Quar. & Plag. & $\mathrm{K}-\mathrm{Fe}$. & Quar. & Mont. & Mica \\
\hline
\end{tabular}


TABLE 3

Summary of X-Ray Mineralogy Samples, Sample Depths, Lithology, Age, and X-Ray Diffraction Results, Site 266

\begin{tabular}{|c|c|c|c|c|c|c|c|c|c|c|c|c|}
\hline \multirow{2}{*}{$\begin{array}{c}\text { Sample } \\
\text { (Interval in } \mathrm{cm} \text { ) }\end{array}$} & \multirow{2}{*}{$\begin{array}{c}\text { Sample } \\
\text { Depth Below } \\
\text { Sea Floor (m) }\end{array}$} & \multirow[b]{2}{*}{ Lithology } & \multirow[b]{2}{*}{ Age } & \multicolumn{3}{|c|}{$\begin{array}{c}\text { Bulk Sample } \\
\text { Major Constituent }\end{array}$} & \multicolumn{3}{|c|}{$\begin{array}{c}2-20 \mu \mathrm{m} \text { Fraction } \\
\text { Major Constituent }\end{array}$} & \multicolumn{3}{|c|}{$\begin{array}{l}<2 \mu \mathrm{m} \text { Fraction } \\
\text { Major Constituent }\end{array}$} \\
\hline & & & & 1 & 2 & 3 & 1 & 2 & 3 & 1 & 2 & 3 \\
\hline $\begin{array}{l}2-3,95-97 \\
5-5,101-103 \\
6-4,70-72 \\
8-3,60-62 \\
9-3,54-56\end{array}$ & $\begin{array}{r}29.0 \\
89.0 \\
106.2 \\
133.1 \\
142.5\end{array}$ & $\begin{array}{c}\text { Unit } 1 \\
\text { Diatom ooze; } \\
\text { minor micarb-bearing } \\
\text { and clay diatom } \\
\text { oozes }\end{array}$ & $\begin{array}{l}\text { Pliocene } \\
\text { and } \\
\text { Pleistocene }\end{array}$ & $\begin{array}{l}\text { Plag. } \\
\text { Mica } \\
\text { Mica } \\
\text { Mica } \\
\text { Calc. }\end{array}$ & $\begin{array}{l}\text { K-Fe. } \\
\text { Plag. } \\
\text { Quar. } \\
\text { Plag. } \\
\text { Mica }\end{array}$ & $\begin{array}{l}\text { Mica } \\
\text { Quar. } \\
\text { Plag. } \\
\text { Quar. } \\
\text { Quar. }\end{array}$ & $\begin{array}{l}\text { Plag. } \\
\text { Plag. } \\
\text { Quar. } \\
\text { Plag. } \\
\text { Quar. }\end{array}$ & $\begin{array}{l}\text { K-Fe. } \\
\text { Quar. } \\
\text { Plag. } \\
\text { Quar. } \\
\text { Plag. }\end{array}$ & $\begin{array}{l}\text { Augi. } \\
\text { K-Fe. } \\
\text { K-Fe. } \\
\text { K-Fe. } \\
\text { K-Fe. }\end{array}$ & $\begin{array}{l}\text { Mont. } \\
\text { Mont. } \\
\text { Mica } \\
\text { Mont. } \\
\text { Quar. }\end{array}$ & $\begin{array}{l}\text { Plag. } \\
\text { Mica } \\
\text { Mont. } \\
\text { Quar. } \\
\text { Mica }\end{array}$ & $\begin{array}{l}\text { K-Fe. } \\
\text { Quar. } \\
\text { Quar. } \\
\text { Mica } \\
\text { Plag. }\end{array}$ \\
\hline $\begin{array}{l}10-4,82-84 \\
10-5,110-112 \\
15-3,92-94 \\
16-2,120-122\end{array}$ & $\begin{array}{l}153.8 \\
155.6 \\
237.9 \\
246.2\end{array}$ & $\begin{array}{c}\text { Unit } 2 \\
\text { Mixed nanno oozes, } \\
\text { nanno clay, diatom } \\
\text { ooze, and diatom- } \\
\text { rich clay }\end{array}$ & $\begin{array}{c}\text { Middle } \\
\text { and } \\
\text { upper } \\
\text { Miocene }\end{array}$ & $\begin{array}{l}\text { Calc. } \\
\text { Calc. } \\
\text { Calc. } \\
\text { Quar. }\end{array}$ & $\begin{array}{l}\text { Mica } \\
\text { Quar. } \\
\text { Mica }\end{array}$ & $\begin{array}{l}\text { Quar. } \\
\text { Mica } \\
\text { Calc. }\end{array}$ & $\begin{array}{l}\text { Quar. } \\
\text { Quar. } \\
\text { Quar. } \\
\text { Quar. }\end{array}$ & $\begin{array}{l}\text { Plag. } \\
\text { Plag. } \\
\text { Mica } \\
\text { Plag. }\end{array}$ & $\begin{array}{l}\text { Mica } \\
\text { Mica } \\
\text { Plag. } \\
\text { Mica }\end{array}$ & $\begin{array}{l}\text { Quar. } \\
\text { Quar. } \\
\text { Quar. } \\
\text { Quar. }\end{array}$ & $\begin{array}{l}\text { Mica } \\
\text { Mica } \\
\text { Mica } \\
\text { Mica }\end{array}$ & $\begin{array}{l}\text { Plag. } \\
\text { Plag. } \\
\text { Plag. } \\
\text { Plag. }\end{array}$ \\
\hline $\begin{array}{l}17-5,85-87 \\
21-3,110-112\end{array}$ & $\begin{array}{l}259.9 \\
333.1\end{array}$ & $\begin{array}{c}\text { Unit } 3 \\
\text { Nanno chalk, } \\
\text { minor ooze, and } \\
\text { nanno claystone }\end{array}$ & $\begin{array}{c}\text { Early } \\
\text { Miocene }\end{array}$ & $\begin{array}{l}\text { Calc. } \\
\text { Calc. }\end{array}$ & Mica & Quar. & $\begin{array}{l}\text { Quar. } \\
\text { Quar. }\end{array}$ & $\begin{array}{l}\text { Plag. } \\
\text { Mica }\end{array}$ & $\begin{array}{l}\text { K-Fe. } \\
\text { K-Fe. }\end{array}$ & $\begin{array}{l}\text { Mica } \\
\text { Quar. }\end{array}$ & $\begin{array}{l}\text { Quar. } \\
\text { Mica }\end{array}$ & $\begin{array}{l}\text { Plag. } \\
\text { Plag. }\end{array}$ \\
\hline
\end{tabular}


TABLE 4

Summary of X-Ray Mineralogy Samples, Sample Depths, Lithology, Age, and X-Ray Diffraction Results, Site 267

\begin{tabular}{|c|c|c|c|c|c|c|c|c|c|c|c|c|}
\hline \multirow{2}{*}{$\begin{array}{c}\text { Sample } \\
\text { (Interval in } \mathrm{cm} \text { ) }\end{array}$} & \multirow{2}{*}{$\begin{array}{c}\text { Sample } \\
\text { Depth Below } \\
\text { Sea Floor (m) }\end{array}$} & \multirow[b]{2}{*}{ Lithology } & \multirow[b]{2}{*}{ Age } & \multicolumn{3}{|c|}{$\begin{array}{c}\text { Bulk Sample } \\
\text { Major Constituent }\end{array}$} & \multicolumn{3}{|c|}{$\begin{array}{c}2-20 \mu \mathrm{m} \text { Fraction } \\
\text { Major Constituent }\end{array}$} & \multicolumn{3}{|c|}{$\begin{array}{l}<2 \mu \mathrm{m} \text { Fraction } \\
\text { Major Constituent }\end{array}$} \\
\hline & & & & 1 & 2 & 3 & 1 & 2 & 3 & 1 & 2 & 3 \\
\hline \multicolumn{13}{|l|}{ Hole 267} \\
\hline $3-4,78-80$ & 94.8 & Unit $1^{\mathrm{a}}$ & a & Mica & Quar. & Plag. & Quar. & Mica & Plag. & Quar. & Mica & Plag. \\
\hline $4-4,77-79$ & 132.8 & Unit $2^{b}$ & b & Calc. & Quar. & Mica & Quar. & Mica & Plag. & Quar. & Mica & Plag. \\
\hline \multicolumn{13}{|l|}{ Hole 267A } \\
\hline $\begin{array}{l}1-6,30-32 \\
3-2,100-102\end{array}$ & $\begin{array}{l}11.8 \\
63.5\end{array}$ & Unit $1^{\mathrm{a}}$ & a & $\begin{array}{l}\text { Mica } \\
\text { Mica }\end{array}$ & $\begin{array}{l}\text { Quar. } \\
\text { Quar. }\end{array}$ & $\begin{array}{l}\text { Plag. } \\
\text { Plag. }\end{array}$ & $\begin{array}{l}\text { Quar. } \\
\text { Quar. }\end{array}$ & $\begin{array}{l}\text { Plag. } \\
\text { Mica }\end{array}$ & $\begin{array}{l}\text { K-Fe. } \\
\text { Plag. }\end{array}$ & $\begin{array}{l}\text { Mica } \\
\text { Quar. }\end{array}$ & $\begin{array}{l}\text { Quar. } \\
\text { Mica }\end{array}$ & $\begin{array}{l}\text { K-Fe. } \\
\text { Plag. }\end{array}$ \\
\hline \multicolumn{13}{|l|}{ Hole 267B } \\
\hline $1-1,105-107$ & 106.1 & & & Mica & Quar. & Plag. & Quar. & Plag. & $\mathrm{K}-\mathrm{Fe}$. & Mica & Quar. & Plag. \\
\hline $3-2,146-148$ & 146.0 & Unit 1 & Early & Mica & Quar. & Plag. & Quar. & Mica & Plag. & Quar. & Mica & Plag. \\
\hline $4-2,102-104$ & 164.5 & Clay and silty & Miocene & Mica & Quar. & Plag. & Quar. & Mica & Plag. & Mica & Quar. & Plag. \\
\hline $6-6,40-42$ & 217.4 & clay diatom ooze, & through & Mica & Quar. & Plag. & Quar. & Mica & Plag. & Mica & Quar. & Plag. \\
\hline $7-6,56-58$ & 246.1 & and mixed clay & Early & Mica & Quar. & Plag. & Quar. & Mica & Plag. & Mica & Quar. & Mont. \\
\hline $8-5,78-80$ & 273.3 & diatom sediments & Pliocene & Quar. & Mica & Plag. & Quar. & Plag. & Mica & Mica & Quar. & Plag. \\
\hline $9-5,110-112$ & 302.1 & & & Quar. & Mica & Plag. & Quar. & Mica & Plag. & Quar. & Mica & Plag. \\
\hline
\end{tabular}

${ }^{a}$ Unit 1 in Hole 267 and Hole 267A consists of clay and silty clay, clay diatom ooze, and intermediate mixed clay diatom sediments. Unit 1 is early Miocene through Quaternary in age.

b Unit 2 in Hole 267 consists of nanno ooze and chalk and micritic limestone. Unit 2 is middle Oligocene through early Miocene in age.

TABLE 5

Summary of X-Ray Mineralogy Samples, Sample Depths, Lithology, Age, and X-Ray Diffraction Results, Site 268

\begin{tabular}{|c|c|c|c|c|c|c|c|c|c|c|c|c|}
\hline \multirow{2}{*}{$\begin{array}{c}\text { Sample } \\
\text { (Interval in } \mathrm{cm} \text { ) }\end{array}$} & \multirow{2}{*}{$\begin{array}{c}\text { Sample } \\
\text { Depth Below } \\
\text { Sea Floor }(\mathrm{m})\end{array}$} & \multirow[b]{2}{*}{ Lithology } & \multirow[b]{2}{*}{ Age } & \multicolumn{3}{|c|}{$\begin{array}{c}\text { Bulk Sample } \\
\text { Major Constituent }\end{array}$} & \multicolumn{3}{|c|}{ 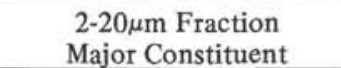 } & \multicolumn{3}{|c|}{$\begin{array}{l}<2 \mu \mathrm{m} \text { Fraction } \\
\text { Major Constituent }\end{array}$} \\
\hline & & & & 1 & 2 & 3 & 1 & 2 & 3 & 1 & 2 & 3 \\
\hline $\begin{array}{l}2-4,57-59 \\
2-4,73-75\end{array}$ & $\begin{array}{l}33.1 \\
33.2\end{array}$ & $\begin{array}{c}\text { Unit } 1 \\
\text { Clay, silty clay, } \\
\text { sand, and diatom } \\
\text { ooze }\end{array}$ & $\begin{array}{c}\text { Late } \\
\text { Miocene } \\
\text { through } \\
\text { Pleistocene }\end{array}$ & $\begin{array}{l}\text { Quar. } \\
\text { Quar. }\end{array}$ & $\begin{array}{l}\text { Mica } \\
\text { Mica }\end{array}$ & $\begin{array}{l}\text { K-Fe. } \\
\text { Plag. }\end{array}$ & $\begin{array}{l}\text { Quar. } \\
\text { Quar. }\end{array}$ & $\begin{array}{l}\text { K-Fe. } \\
\text { Plag. }\end{array}$ & $\begin{array}{l}\text { Plag. } \\
\text { K-Fe. }\end{array}$ & $\begin{array}{l}\text { Mica } \\
\text { Mica }\end{array}$ & $\begin{array}{l}\text { Quar. } \\
\text { Quar. }\end{array}$ & $\begin{array}{l}\text { K-Fe. } \\
\text { Plag. }\end{array}$ \\
\hline $\begin{array}{l}8-1,45-47 \\
8-1,71-73\end{array}$ & $\begin{array}{l}170.9 \\
171.2\end{array}$ & $\begin{array}{c}\text { Unit } 2 \\
\text { Clay, silty clay, } \\
\text { and clay nanno ooze }\end{array}$ & $\begin{array}{c}\text { Late } \\
\text { Miocene }\end{array}$ & $\begin{array}{l}\text { Quar. } \\
\text { Quar. }\end{array}$ & $\begin{array}{l}\text { Mica } \\
\text { Mica }\end{array}$ & $\begin{array}{l}\text { Plag. } \\
\text { Plag. }\end{array}$ & $\begin{array}{l}\text { Quar. } \\
\text { Quar. }\end{array}$ & $\begin{array}{l}\text { Plag. } \\
\text { Plag. }\end{array}$ & $\begin{array}{l}\text { K-Fe. } \\
\text { Mica }\end{array}$ & $\begin{array}{l}\text { Mica } \\
\text { Mica }\end{array}$ & $\begin{array}{l}\text { Quar. } \\
\text { Quar. }\end{array}$ & $\begin{array}{l}\text { Plag. } \\
\text { Plag. }\end{array}$ \\
\hline $\begin{array}{l}10-2,40-42 \\
13-1,80-82\end{array}$ & $\begin{array}{l}229.4 \\
304.3\end{array}$ & $\begin{array}{c}\text { Unit } 3 \\
\text { Silty clay, }\end{array}$ & Late & $\begin{array}{l}\text { Quar. } \\
\text { Cris. }\end{array}$ & $\begin{array}{l}\text { Mica } \\
\text { Quar. }\end{array}$ & $\begin{array}{l}\text { K-Fe. } \\
\text { Mica }\end{array}$ & $\begin{array}{l}\text { Quar. } \\
\text { Ins }\end{array}$ & $\begin{array}{l}\text { Plag. } \\
\text { ficient } r\end{array}$ & $\begin{array}{l}\text { K-Fe. } \\
\text { due }\end{array}$ & $\begin{array}{l}\text { Mica } \\
\text { In }\end{array}$ & $\begin{array}{l}\text { Quar. } \\
\text { ficient r }\end{array}$ & $\begin{array}{l}\text { Plag. } \\
\text { due }\end{array}$ \\
\hline $13-1,75-77$ & 304.3 & laminated silty clay & Miocene & Mica & Quar. & Plag. & Quar. & Mica & Plag. & Mica & Quar. & Plag. \\
\hline $13-1,98-100$ & 304.5 & and clayey silt, & or & Quar. & $\mathrm{K}-\mathrm{Fe}$. & Plag. & Quar. & Plag. & $\mathrm{K}$-Fe. & Mica & Quar. & Plag. \\
\hline $17-1,140-142$ & 380.9 & and chert & older & Quar. & Mica & $\mathrm{K}-\mathrm{Fe}$. & Quar. & $\mathrm{K}-\mathrm{Fe}$. & Plag. & Quar. & Mica & $\mathrm{K}-\mathrm{Fe}$. \\
\hline $20-2,83-84$ & 467.3 & & & Quar. & Mica & $\mathrm{K}-\mathrm{Fe}$. & Quar. & $\mathrm{K}-\mathrm{Fe}$. & Plag. & Quar. & Mica & $\mathrm{K}-\mathrm{Fe}$. \\
\hline
\end{tabular}


TABLE 6

Summary of X-Ray Mineralogy Samples, Sample Depths, Lithology, Age, and X-Ray Diffraction Results, Site 269

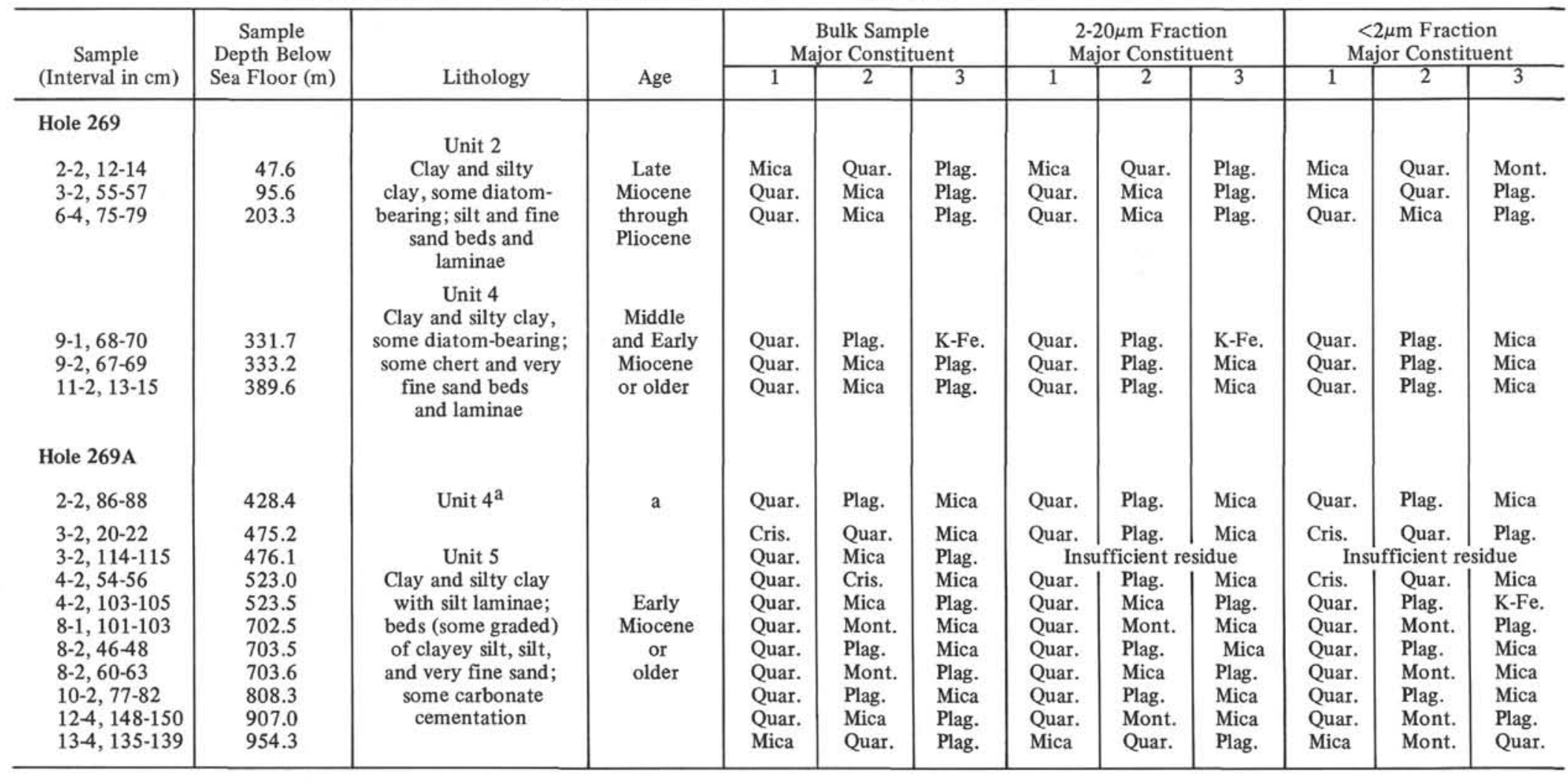

${ }^{\mathrm{a}}$ Unit 4 in Hole $269 \mathrm{~A}$ is identical to Unit 4 in Hole 269 , and is middle and early Miocene or older. 
TABLE 7

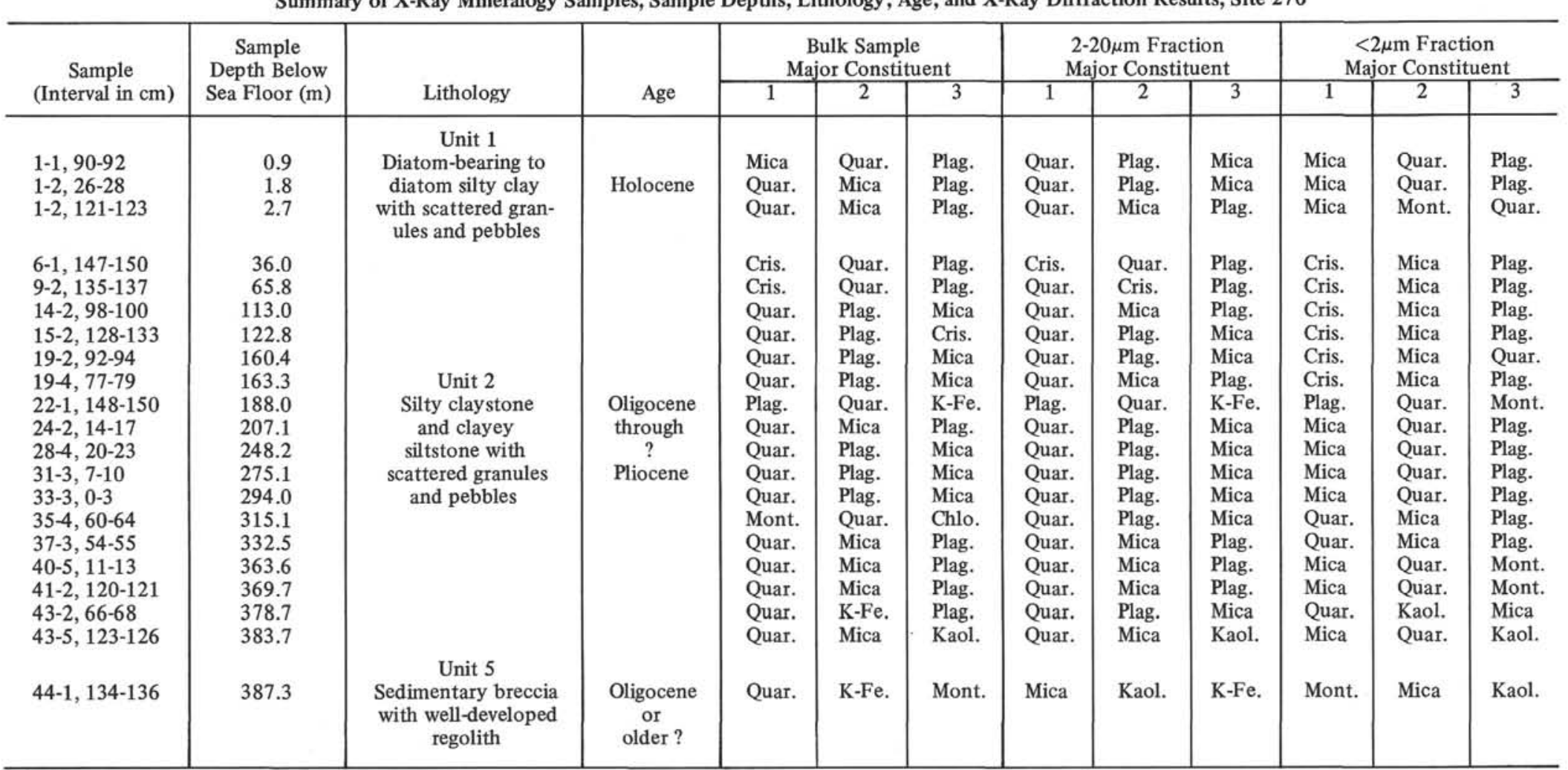

TABLE 8

Summary of X-Ray Mineralogy Samples, Sample Depths, Lithology, Age, and X-Ray Diffraction Results, Site 27

\begin{tabular}{|c|c|c|c|c|c|c|c|c|c|c|c|c|}
\hline \multirow{2}{*}{$\begin{array}{c}\text { Sample } \\
\text { (Interval in } \mathrm{cm} \text { ) }\end{array}$} & \multirow{2}{*}{$\begin{array}{c}\text { Sample } \\
\text { Depth Below } \\
\text { Sea Floor (m) }\end{array}$} & \multirow[b]{2}{*}{ Lithology } & \multirow[b]{2}{*}{ Age } & \multicolumn{3}{|c|}{$\begin{array}{c}\text { Bulk Sample } \\
\text { Major Constituent }\end{array}$} & \multicolumn{3}{|c|}{ 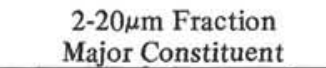 } & \multicolumn{3}{|c|}{$\begin{array}{c}<2 \mu \mathrm{m} \text { Fraction } \\
\text { Major Constituent }\end{array}$} \\
\hline & & & & 1 & 2 & 3 & 1 & 2 & 3 & 1 & 2 & 3 \\
\hline $\begin{array}{l}5-1,107-109 \\
24-2,128-130\end{array}$ & $\begin{array}{r}69.6 \\
158.3\end{array}$ & $\begin{array}{c}\text { Unit } 1 \\
\text { Clayey silt and } \\
\text { silty clays with } \\
\text { scattered granules } \\
\text { and larger clasts }\end{array}$ & Pliocene & $\begin{array}{l}\text { Quar. } \\
\text { Mica }\end{array}$ & $\begin{array}{l}\text { Mica } \\
\text { Quar. }\end{array}$ & $\begin{array}{l}\text { Plag. } \\
\text { Plag. }\end{array}$ & $\begin{array}{l}\text { Quar. } \\
\text { Quar. }\end{array}$ & $\begin{array}{l}\text { Plag. } \\
\text { Plag. }\end{array}$ & $\begin{array}{l}\text { Mica } \\
\text { Mica }\end{array}$ & $\begin{array}{l}\text { Chlo. } \\
\text { Mica }\end{array}$ & $\begin{array}{l}\text { Mica } \\
\text { Plag. }\end{array}$ & $\begin{array}{l}\text { Quar. } \\
\text { Mont. }\end{array}$ \\
\hline
\end{tabular}


TABLE 9

Summary of X-Ray Mineralogy Samples, Sample Depths, Lithology, Age, and X-Ray Diffraction Results, Site 272

\begin{tabular}{|c|c|c|c|c|c|c|c|c|c|c|c|c|}
\hline \multirow{2}{*}{$\begin{array}{c}\text { Sample } \\
\text { (Interval in } \mathrm{cm} \text { ) }\end{array}$} & \multirow{2}{*}{$\begin{array}{c}\text { Sample } \\
\text { Depth Below } \\
\text { Sea Floor }(\mathrm{m})\end{array}$} & \multirow[b]{2}{*}{ Lithology } & \multirow[b]{2}{*}{ Age } & \multicolumn{3}{|c|}{$\begin{array}{c}\text { Bulk Sample } \\
\text { Major Constituent }\end{array}$} & \multicolumn{3}{|c|}{ 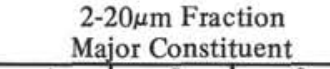 } & \multicolumn{3}{|c|}{$\begin{array}{c}<2 \mu \mathrm{m} \text { Fraction } \\
\text { Major Constituent }\end{array}$} \\
\hline & & & & 1 & 2 & 3 & 1 & 2 & 3 & 1 & 2 & 3 \\
\hline $\begin{array}{l}1-1,60-62 \\
1-3,79-81\end{array}$ & $\begin{array}{l}4.6 \\
7.8\end{array}$ & $\begin{array}{c}\text { Unit 1B } \\
\text { Diatom-bearing silty } \\
\text { clay with scattered } \\
\text { granules and } \\
\text { larger clasts }\end{array}$ & Pleistocene & $\begin{array}{l}\text { Mica } \\
\text { Quar. }\end{array}$ & $\begin{array}{l}\text { Quar. } \\
\text { Mica }\end{array}$ & $\begin{array}{l}\text { Plag. } \\
\text { Plag. }\end{array}$ & $\begin{array}{l}\text { Quar. } \\
\text { Quar. }\end{array}$ & $\begin{array}{l}\text { Mica } \\
\text { Plag. }\end{array}$ & $\begin{array}{l}\text { Plag. } \\
\text { Mica }\end{array}$ & $\begin{array}{l}\text { Mica } \\
\text { Mica }\end{array}$ & $\begin{array}{l}\text { Quar. } \\
\text { Mont. }\end{array}$ & $\begin{array}{l}\text { Plag. } \\
\text { Quar. }\end{array}$ \\
\hline $\begin{array}{l}9-3,134-136 \\
12-1,107-109 \\
15-2,103-105\end{array}$ & $\begin{array}{r}84.3 \\
109.6 \\
139.5\end{array}$ & $\begin{array}{c}\text { Unit } 2 \mathrm{~A} \\
\text { Silty claystone with } \\
\text { scattered clasts }\end{array}$ & $\begin{array}{c}\text { Late } \\
\text { Miocene? }\end{array}$ & $\begin{array}{l}\text { Quar. } \\
\text { Quar. } \\
\text { Quar. }\end{array}$ & $\begin{array}{l}\text { Mica } \\
\text { Mica } \\
\text { Mica }\end{array}$ & $\begin{array}{l}\text { Plag. } \\
\text { Plag. } \\
\text { Plag. }\end{array}$ & $\begin{array}{l}\text { Quar. } \\
\text { Quar. } \\
\text { Quar. }\end{array}$ & $\begin{array}{l}\text { Mica } \\
\text { Mica } \\
\text { Plag. }\end{array}$ & $\begin{array}{l}\text { Plag. } \\
\text { Plag. } \\
\text { Mica }\end{array}$ & $\begin{array}{l}\text { Mica } \\
\text { Mica } \\
\text { Mica }\end{array}$ & $\begin{array}{l}\text { Plag. } \\
\text { Plag. } \\
\text { Plag. }\end{array}$ & $\begin{array}{l}\text { Quar. } \\
\text { Quar. } \\
\text { Quar. }\end{array}$ \\
\hline $\begin{array}{l}19-6,76-78 \\
22-3,58-60 \\
30-2,24-26 \\
38-2,83-85\end{array}$ & $\begin{array}{l}183.3 \\
207.1 \\
281.2 \\
357.8\end{array}$ & $\begin{array}{c}\text { Unit 2B } \\
\text { Diatom silty claystone } \\
\text { with silty clay } \\
\text { diatomite rare clasts }\end{array}$ & $\begin{array}{l}\text { Middle } \\
\text { Miocene }\end{array}$ & $\begin{array}{l}\text { Quar. } \\
\text { Quar. } \\
\text { Quar. } \\
\text { Quar. }\end{array}$ & $\begin{array}{l}\text { Plag. } \\
\text { Plag. } \\
\text { Plag. } \\
\text { Plag. }\end{array}$ & $\begin{array}{l}\text { Mica } \\
\text { Mica } \\
\text { K-Fe. } \\
\text { Mica }\end{array}$ & $\begin{array}{l}\text { Quar. } \\
\text { Quar. } \\
\text { Quar. } \\
\text { Quar. }\end{array}$ & $\begin{array}{l}\text { Plag. } \\
\text { Plag. } \\
\text { Plag. } \\
\text { Plag. }\end{array}$ & $\begin{array}{l}\text { Mica } \\
\text { Mica } \\
\text { Mica } \\
\text { Mica }\end{array}$ & $\begin{array}{l}\text { Mica } \\
\text { Mica } \\
\text { Plag. } \\
\text { Cris. }\end{array}$ & $\begin{array}{l}\text { Plag. } \\
\text { Plag. } \\
\text { Mica } \\
\text { Mica }\end{array}$ & $\begin{array}{l}\text { Quar. } \\
\text { Quar. } \\
\text { Quar. } \\
\text { Plag. }\end{array}$ \\
\hline $\begin{array}{l}41-1,50-51 \\
41-1,146-148\end{array}$ & $\begin{array}{l}384.5 \\
385.5\end{array}$ & $\begin{array}{c}\text { Unit 2C } \\
\text { Silty claystone } \\
\text { with rare clasts }\end{array}$ & $\begin{array}{l}\text { Middle } \\
\text { and early }\end{array}$ & $\begin{array}{l}\text { Cris. } \\
\text { Cris. }\end{array}$ & $\begin{array}{l}\text { Quar. } \\
\text { Quar. }\end{array}$ & $\begin{array}{l}\text { Plag. } \\
\text { Plag. }\end{array}$ & $\begin{array}{l}\text { Quar. } \\
\text { Ins }\end{array}$ & $\begin{array}{l}\text { Plag. } \\
\text { ficient }\end{array}$ & $\begin{array}{l}\text { Mica } \\
\text { idue }\end{array}$ & $\begin{array}{l}\text { Cris. } \\
\text { In }\end{array}$ & $\begin{array}{l}\text { Mica } \\
\text { ficient }\end{array}$ & $\begin{array}{l}\text { Plag. } \\
\text { due }\end{array}$ \\
\hline
\end{tabular}

TABLE 10

Summary of X-Ray Mineralogy Samples, Sample Depths, Lithology, Age, and X-Ray Diffraction Results, Site 273

\begin{tabular}{|c|c|c|c|c|c|c|c|c|c|c|c|c|}
\hline \multirow{2}{*}{$\begin{array}{c}\text { Sample } \\
\text { (Interval in } \mathrm{cm} \text { ) }\end{array}$} & \multirow{2}{*}{$\begin{array}{c}\text { Sample } \\
\text { Depth Below } \\
\text { Sea Floor }(\mathrm{m})\end{array}$} & \multirow[b]{2}{*}{ Lithology } & \multirow[b]{2}{*}{ Age } & \multicolumn{3}{|c|}{$\begin{array}{c}\text { Bulk Sample } \\
\text { Major Constituent }\end{array}$} & \multicolumn{3}{|c|}{ 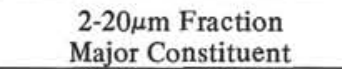 } & \multicolumn{3}{|c|}{$\begin{array}{c}<2 \mu \mathrm{m} \text { Fraction } \\
\text { Major Constituent }\end{array}$} \\
\hline & & & & 1 & 2 & 3 & 1 & 2 & 3 & 1 & 2 & 3 \\
\hline $\begin{array}{l}\text { Hole } 273 \\
2-4,90-92\end{array}$ & 9.9 & $\begin{array}{c}\text { Unit 1B } \\
\text { Diatom-bearing } \\
\text { pebbly silty clay, }\end{array}$ & Pliocene & Quar. & Mica & Plag. & \multicolumn{3}{|c|}{ Insufficient residue } & Mica & Quar. & Plag. \\
\hline $4-2,110-112$ & 26.1 & unbedded & & Quar. & Plag. & Mica & Quar. & Plag. & Mica & Mica & Quar. & Plag. \\
\hline $6-2,55-57$ & 44.5 & Unit $2 A^{a}$ & a & Quar. & Plag. & Mica & Quar. & Plag. & Mica & Mica & Quar. & Plag. \\
\hline Hole 273A & & & & & & & & & & & & \\
\hline $\begin{array}{l}6-2,85-87 \\
7-2,51-55 \\
7-2,104-108 \\
9-1,91-95\end{array}$ & $\begin{array}{l}139.9 \\
149.0 \\
149.5 \\
166.9\end{array}$ & Unit $2 A^{a}$ & a & $\begin{array}{l}\text { Quar. } \\
\text { Dolo. } \\
\text { Quar. } \\
\text { Quar. }\end{array}$ & $\begin{array}{l}\text { Mica } \\
\text { Quar. } \\
\text { Plag. } \\
\text { Plag. }\end{array}$ & $\begin{array}{l}\text { Plag. } \\
\text { Plag. } \\
\text { Mica } \\
\text { K-Fe. }\end{array}$ & $\begin{array}{l}\text { Quar. } \\
\text { Quar. } \\
\text { Quar. } \\
\text { Quar. }\end{array}$ & $\begin{array}{l}\text { Plag. } \\
\text { Plag. } \\
\text { Plag. } \\
\text { Plag. }\end{array}$ & $\begin{array}{l}\text { K-Fe. } \\
\text { K-Fe. } \\
\text { Mica } \\
\text { K-Fe. }\end{array}$ & $\begin{array}{l}\text { Quar. } \\
\text { Quar. } \\
\text { Plag. } \\
\text { Mica }\end{array}$ & $\begin{array}{l}\text { Mica } \\
\text { Plag. } \\
\text { Quar. } \\
\text { Quar. }\end{array}$ & $\begin{array}{l}\text { Plag. } \\
\text { Mica } \\
\text { Mica } \\
\text { Plag. }\end{array}$ \\
\hline $\begin{array}{l}13-3,127-130 \\
17-2,79-82 \\
22-1,78-81 \\
25-2,112-115\end{array}$ & $\begin{array}{l}198.8 \\
234.8 \\
273.3 \\
301.6\end{array}$ & $\begin{array}{c}\text { Unit 2B } \\
\text { Pebbly silty clay, } \\
\text { some diatom-bearing, } \\
\text { unbedded }\end{array}$ & & $\begin{array}{l}\text { Quar. } \\
\text { Quar. } \\
\text { Quar. } \\
\text { Quar. }\end{array}$ & $\begin{array}{l}\text { Plag. } \\
\text { Plag. } \\
\text { K-Fe. } \\
\text { Plag. }\end{array}$ & $\begin{array}{l}\text { Mica } \\
\text { Mica } \\
\text { Plag. } \\
\text { Mica }\end{array}$ & $\begin{array}{l}\text { Quar. } \\
\text { Quar. } \\
\text { Quar. } \\
\text { Quar. }\end{array}$ & $\begin{array}{l}\text { Plag. } \\
\text { Plag. } \\
\text { Plag. } \\
\text { Plag. }\end{array}$ & $\begin{array}{l}\text { Mica } \\
\text { Mica } \\
\text { Mica } \\
\text { Mica }\end{array}$ & $\begin{array}{l}\text { Mica } \\
\text { Mica } \\
\text { Mica } \\
\text { Mica }\end{array}$ & $\begin{array}{l}\text { Quar. } \\
\text { Quar. } \\
\text { Plag. } \\
\text { Plag. }\end{array}$ & $\begin{array}{l}\text { Plag. } \\
\text { Plag. } \\
\text { Quar. } \\
\text { Quar. }\end{array}$ \\
\hline
\end{tabular}

${ }^{\mathrm{a}}$ Unit $2 \mathrm{~A}$ in Hole 273 and Hole 273A consists of pebbly silty clay, diatom-bearing, sparsely bedded. Unit 2A is middle and late Miocene in age. 


\begin{tabular}{|c|c|c|c|c|c|c|c|c|c|c|c|c|}
\hline \multirow{2}{*}{$\begin{array}{c}\text { Sample } \\
\text { (Interval in } \mathrm{cm} \text { ) }\end{array}$} & \multirow{2}{*}{$\begin{array}{c}\text { Sample } \\
\text { Depth Below } \\
\text { Sea Floor }(\mathrm{m})\end{array}$} & \multirow[b]{2}{*}{ Lithology } & \multirow[b]{2}{*}{ Age } & \multicolumn{3}{|c|}{$\begin{array}{c}\text { Bulk Sample } \\
\text { Major Constituent }\end{array}$} & \multicolumn{3}{|c|}{ 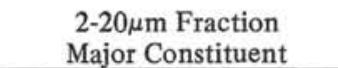 } & \multicolumn{3}{|c|}{$\begin{array}{l}<2 \mu \mathrm{m} \text { Fraction } \\
\text { Major Constituent }\end{array}$} \\
\hline & & & & 1 & 2 & 3 & 1 & 2 & 3 & 1 & 2 & 3 \\
\hline $\begin{array}{l}1-4,120-122 \\
2-3,68-70 \\
5-3,130-132 \\
6-2,130-132\end{array}$ & $\begin{array}{r}5.7 \\
13.2 \\
42.3 \\
50.3\end{array}$ & $\begin{array}{l}\text { Unit } 1 \\
\text { Diatom-rich silty } \\
\text { clay with pebbles }\end{array}$ & $\begin{array}{c}\text { Early } \\
\text { Pliocene } \\
\text { through } \\
\text { Pleistocene }\end{array}$ & $\begin{array}{l}\text { Mica } \\
\text { Mica } \\
\text { Mica } \\
\text { Mica }\end{array}$ & $\begin{array}{l}\text { Quar. } \\
\text { Quar. } \\
\text { Quar. } \\
\text { Quar. }\end{array}$ & $\begin{array}{l}\text { Plag. } \\
\text { Plag. } \\
\text { Plag. } \\
\text { Plag. }\end{array}$ & $\begin{array}{l}\text { Quar. } \\
\text { Quar. } \\
\text { Quar. } \\
\text { Quar. }\end{array}$ & $\begin{array}{l}\text { Plag. } \\
\text { Plag. } \\
\text { Plag. } \\
\text { Plag. }\end{array}$ & $\begin{array}{l}\text { Mica } \\
\text { Mica } \\
\text { Mica } \\
\text { Mica }\end{array}$ & $\begin{array}{l}\text { Mica } \\
\text { Mica } \\
\text { Mica } \\
\text { Mica }\end{array}$ & $\begin{array}{l}\text { Mont. } \\
\text { Mont. } \\
\text { Mont. } \\
\text { Mont. }\end{array}$ & $\begin{array}{l}\text { Quar. } \\
\text { Quar. } \\
\text { Quar. } \\
\text { Quar. }\end{array}$ \\
\hline $\begin{array}{l}10-3,90-92 \\
13-2,110-112 \\
13-6,110-112\end{array}$ & $\begin{array}{r}89.4 \\
116.6 \\
122.6\end{array}$ & $\begin{array}{c}\text { Unit } 2 \\
\text { Diatom detrital silty } \\
\text { clay with pebbles and } \\
\text { manganese nodules }\end{array}$ & $\begin{array}{l}\text { Middle } \\
\text { Miocene } \\
\text { and early } \\
\text { Pliocene }\end{array}$ & $\begin{array}{l}\text { Quar. } \\
\text { Mica } \\
\text { Quar. }\end{array}$ & $\begin{array}{l}\text { Mica } \\
\text { Quar. } \\
\text { Mica }\end{array}$ & $\begin{array}{l}\text { Plag. } \\
\text { Plag. } \\
\text { Plag. }\end{array}$ & $\begin{array}{l}\text { Quar. } \\
\text { Quar. } \\
\text { Quar. }\end{array}$ & $\begin{array}{l}\text { Mica } \\
\text { Plag. } \\
\text { Plag. }\end{array}$ & $\begin{array}{l}\text { Plag. } \\
\text { Mica } \\
\text { Mica }\end{array}$ & $\begin{array}{l}\text { Mica } \\
\text { Mica } \\
\text { Mont. }\end{array}$ & $\begin{array}{l}\text { Mont. } \\
\text { Mont. } \\
\text { Mica }\end{array}$ & $\begin{array}{l}\text { Quar. } \\
\text { Quar. } \\
\text { Quar. }\end{array}$ \\
\hline $\begin{array}{l}14-2,90-92 \\
17-2,82-84\end{array}$ & $\begin{array}{l}125.9 \\
154.3\end{array}$ & $\begin{array}{c}\text { Unit } 3 \\
\text { Diatom-rich } \\
\text { silty clay }\end{array}$ & $\begin{array}{l}\text { ? Early } \\
\text { and middle } \\
\text { Miocene }\end{array}$ & $\begin{array}{l}\text { Quar. } \\
\text { Mica }\end{array}$ & $\begin{array}{l}\text { Mica } \\
\text { Quar. }\end{array}$ & $\begin{array}{l}\text { Plag. } \\
\text { Plag. }\end{array}$ & $\begin{array}{l}\text { Quar. } \\
\text { Quar. }\end{array}$ & $\begin{array}{l}\text { Plag. } \\
\text { Plag. }\end{array}$ & $\begin{array}{l}\text { Mica } \\
\text { Mica }\end{array}$ & $\begin{array}{l}\text { Mica } \\
\text { Mica }\end{array}$ & $\begin{array}{l}\text { Plag. } \\
\text { Quar. }\end{array}$ & $\begin{array}{l}\text { Quar. } \\
\text { Plag. }\end{array}$ \\
\hline $\begin{array}{l}20-3,90-92 \\
21-6,70-72 \\
23-2,140-142 \\
26-3,90-92 \\
29-2,33-35 \\
32-4,140-142\end{array}$ & $\begin{array}{l}184.4 \\
198.2 \\
211.9 \\
241.4 \\
267.8 \\
300.4\end{array}$ & $\begin{array}{c}\text { Unit } 4 \\
\text { Diatom-detrital } \\
\text { silty clay and minor } \\
\text { silty clay diatom } \\
\text { ooze, mostly } \\
\text { non-bedded }\end{array}$ & $\begin{array}{c}\text { Early } \\
\text { and } \\
\text { middle } \\
\text { Oligocene }\end{array}$ & $\begin{array}{l}\text { Mica } \\
\text { Quar. } \\
\text { Quar. } \\
\text { Quar. } \\
\text { Quar. } \\
\text { Quar. }\end{array}$ & $\begin{array}{l}\text { Quar. } \\
\text { Mica } \\
\text { Mica } \\
\text { Mica } \\
\text { Mica } \\
\text { Mica }\end{array}$ & $\begin{array}{l}\text { Plag. } \\
\text { Plag. } \\
\text { Plag. } \\
\text { Plag. } \\
\text { Plag. } \\
\text { Plag. }\end{array}$ & $\begin{array}{l}\text { Quar. } \\
\text { Quar. } \\
\text { Quar. } \\
\text { Quar. } \\
\text { Quar. } \\
\text { Quar. }\end{array}$ & $\begin{array}{l}\text { Plag. } \\
\text { Plag. } \\
\text { Plag. } \\
\text { Plag. } \\
\text { Plag. } \\
\text { Plag. }\end{array}$ & $\begin{array}{l}\text { Mica } \\
\text { Mica } \\
\text { Mica } \\
\text { K-Fe. } \\
\text { K-Fe. } \\
\text { Mica }\end{array}$ & $\begin{array}{l}\text { Mica } \\
\text { Quar. } \\
\text { Quar. } \\
\text { Mica } \\
\text { Mica } \\
\text { Mica }\end{array}$ & $\begin{array}{l}\text { Mont. } \\
\text { Mica } \\
\text { Mica } \\
\text { Quar. } \\
\text { Quar. } \\
\text { Mont. }\end{array}$ & $\begin{array}{l}\text { Quar. } \\
\text { Plag. } \\
\text { Plag. } \\
\text { Plag. } \\
\text { Plag. } \\
\text { Quar. }\end{array}$ \\
\hline $\begin{array}{l}39-2,107-110 \\
42-2,137-139\end{array}$ & $\begin{array}{l}363.6 \\
392.4\end{array}$ & $\begin{array}{c}\text { Unit } 5 \\
\text { Silty claystone } \\
\text { locally chert-bearing }\end{array}$ & $\begin{array}{l}\text { ?Cretaceous } \\
\text { through } \\
\text { Eocene }\end{array}$ & $\begin{array}{l}\text { Quar. } \\
\text { Cris. }\end{array}$ & $\begin{array}{l}\text { Mica } \\
\text { Mica }\end{array}$ & $\begin{array}{l}\text { Cris. } \\
\text { Mont. }\end{array}$ & $\begin{array}{l}\text { Quar. } \\
\text { Cris. }\end{array}$ & $\begin{array}{l}\text { Plag. } \\
\text { Quar. }\end{array}$ & $\begin{array}{l}\text { Mica } \\
\text { Mica }\end{array}$ & $\begin{array}{l}\text { Cris. } \\
\text { Cris. }\end{array}$ & $\begin{array}{l}\text { Quar. } \\
\text { Mont. }\end{array}$ & Mica \\
\hline
\end{tabular}


TABLE 12

Results of X-Ray Diffraction Analy sis From Site 264

\begin{tabular}{|c|c|c|c|c|c|c|c|c|c|c|c|c|c|}
\hline Core & $\begin{array}{c}\text { Cored } \\
\text { Interval } \\
\text { Below Sea } \\
\text { Floor (m) }\end{array}$ & $\begin{array}{l}\text { Sample Depth } \\
\text { Below Sea } \\
\text { Floor }(\mathrm{m})\end{array}$ & 客 & சुँ & हึँ & $\stackrel{\text { aी }}{\pi}$ & 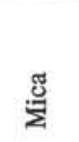 & 范 & 晃 & 音 & 苂 & 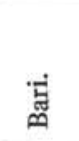 & 茎 \\
\hline \multicolumn{14}{|c|}{ Bulk Samples } \\
\hline 8 & $101.5-111.0$ & 105.1 & - & 97.6 & 0.3 & & - & 1.6 & - & 0.5 & - & - & \\
\hline 10 & $158.5-168.0$ & 161.1 & - & 98.2 & 0.4 & & - & - & - & 1.4 & - & - & \\
\hline & & 161.8 & - & 98.5 & 0.4 & & - & - & - & 1.1 & - & - & \\
\hline & & 162.9 & 2.3 & 77.6 & 0.8 & & 1.3 & - & 1.4 & 11.6 & 5.7 & 1.7 & \\
\hline 11 & $168.0-177.5$ & 169.7 & - & 98.0 & 0.2 & & - & - & - & 1.3 & - & 0.5 & \\
\hline \multicolumn{14}{|c|}{$2 \cdot 20 \mu \mathrm{m}$ Fractions } \\
\hline 8 & $101.5-111.0$ & 105.1 & 20.7 & & 18.9 & - & 17.0 & 24.6 & & 39.5 & & - & \\
\hline 10 & $158.5-168.0$ & 161.1 & 4.7 & & 12.8 & - & 11.8 & 11.0 & & 64.3 & & - & \\
\hline & & 161.8 & 19.6 & & 11.4 & - & 6.4 & - & & 82.2 & & - & \\
\hline & & 162.9 & 7.2 & & 6.4 & - & 3.6 & - & & 85.3 & & 4.7 & \\
\hline 11 & $168.0-177.5$ & 169.7 & 17.1 & & 15.0 & 3.3 & 6.4 & - & & 62.5 & & 12.8 & \\
\hline \multicolumn{14}{|c|}{$<2 \mu \mathrm{m}$ Fractions } \\
\hline 8 & $101.5-111.0$ & 105.1 & 60.5 & & 5.2 & & 3.6 & 80.7 & 5.5 & 2.7 & - & & 2.2 \\
\hline \multirow[t]{3}{*}{10} & $158.5-168.0$ & 161.1 & 49.3 & & 4.1 & & 5.6 & 86.2 & - & 1.9 & - & & 2.3 \\
\hline & & 161.8 & 41.8 & & 3.8 & & 8.8 & 73.9 & 10.0 & 2.1 & - & & 1.4 \\
\hline & & 162.9 & 65.6 & & 2.8 & & - & 12.4 & 14.3 & 6.5 & 56.6 & & 7.3 \\
\hline 11 & $168.0-177.5$ & 169.7 & 57.4 & & 5.1 & & - & 36.2 & 40.7 & 14.3 & - & & 3.6 \\
\hline
\end{tabular}

TABLE 13

Results of X-Ray Diffraction Analy sis From Site 265

\begin{tabular}{|c|c|c|c|c|c|c|c|c|c|c|c|c|c|c|}
\hline Core & $\begin{array}{c}\text { Cored } \\
\text { Interval } \\
\text { Below Sea } \\
\text { Floor }(\mathrm{m})\end{array}$ & $\begin{array}{l}\text { Sample Depth } \\
\text { Below Sea } \\
\text { Floor }(\mathrm{m})\end{array}$ & 客 & गี & हैँ & 这 & $\frac{\text { a }}{2}$ & 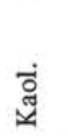 & 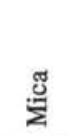 & 范 & 完 & 筥 & 宓 & $\frac{\pi}{5}$ \\
\hline \multicolumn{15}{|c|}{ Bulk Samples } \\
\hline 2 & $18.5-28.0$ & 27.0 & 51.4 & 92.4 & 0.5 & 1.3 & 1.4 & 1.3 & 1.8 & - & - & 1.4 & - & $\mathrm{P}$ \\
\hline & t & 27.2 & 92.0 & - & 16.9 & 17.7 & 28.6 & 5.5 & 11.5 & 19.7 & - & - & - & - \\
\hline 5 & $104.0-113.5$ & 111.3 & 82.6 & - & 11.0 & 11.7 & 24.5 & 3.8 & 10.3 & 25.4 & 1.9 & - & 11.4 & - \\
\hline 7 & $161.0-170.5$ & 166.7 & 65.3 & 99.0 & 1.0 & - & - & - & - & - & - & - & - & $\mathrm{T}$ \\
\hline 14 & $360.5-370.0$ & 369.3 & 92.9 & - & 19.4 & 17.6 & 21.2 & 3.5 & 27.3 & 11.1 & - & - & - & - \\
\hline 15 & $398.5-408.0$ & 403.8 & 19.8 & 98.7 & 1.3 & - & - & - & - & - & - & - & - & $\mathrm{T}$ \\
\hline \multicolumn{15}{|c|}{$2-20 \mu \mathrm{m}$ Fractions } \\
\hline 2 & $18.5-28.0$ & 27.2 & 89.0 & & 13.6 & 19.1 & 26.9 & 3.1 & 7.0 & 10.1 & - & & 20.3 & \\
\hline 5 & $104.0-113.5$ & 111.3 & 82.8 & & 11.6 & 16.5 & 30.4 & 3.0 & 5.3 & 8.7 & 3.6 & & 20.9 & \\
\hline 14 & $360.5-370.0$ & 369.3 & 94.1 & & 23.3 & 38.2 & 27.7 & 2.0 & 8.8 & - & - & & - & \\
\hline 15 & $398.5-408.0$ & 403.8 & 92.2 & & 38.4 & 18.5 & 33.1 & 3.3 & 6.7 & - & - & & - & \\
\hline \multicolumn{15}{|c|}{$<2 \mu \mathrm{m}$ Fractions } \\
\hline 2 & $18.5-28.0$ & 27.2 & 87.7 & & 10.5 & 12.0 & 17.5 & 4.7 & 9.0 & 46.3 & - & & - & \\
\hline 5 & $104.0-113.5$ & 111.3 & 79.4 & & 8.5 & 9.6 & 13.3 & 4.6 & 6.4 & 50.6 & 1.3 & & 5.7 & \\
\hline 14 & $360.5-370.0$ & 369.3 & 92.0 & & 16.1 & 19.0 & 16.8 & 5.6 & 18.6 & 23.9 & - & & - & \\
\hline
\end{tabular}

${ }^{\mathrm{a}} \mathrm{U}-1$ peaks at $5.76 \AA, 3.63 \AA, 2.357 \AA$, among others. 
TABLE 14

Results of X-Ray Diffraction Analysis From Site 266

\begin{tabular}{|c|c|c|c|c|c|c|c|c|c|c|c|c|c|c|c|c|}
\hline Core & $\begin{array}{c}\text { Cored } \\
\text { Interval } \\
\text { Below Sea } \\
\text { Floor (m) }\end{array}$ & $\begin{array}{l}\text { Sample Depth } \\
\text { Below Sea } \\
\text { Floor }(\mathrm{m})\end{array}$ & 它 & गु & 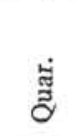 & $\frac{0}{4}$ & 离 & ஓं & 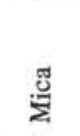 & $\frac{\dot{0}}{0}$ & $\stackrel{\ddot{\Xi}}{\sum}$ & त्ञ & 苟 & 惣 & 官 & 岛 \\
\hline \multicolumn{17}{|c|}{ Bulk Samples } \\
\hline 2 & $25.0-34.5$ & 29.0 & 94.5 & - & 14.4 & 22.0 & 28.4 & - & 16.3 & - & 9.8 & & - & & - & 9.2 \\
\hline 5 & $82.0-91.5$ & 89.0 & 79.4 & - & 17.7 & 15.3 & 17.8 & 4.5 & 28.8 & - & 11.0 & & - & & 1.4 & 3.4 \\
\hline 6 & $101.0-110.5$ & 106.2 & 88.3 & - & 22.7 & 15.4 & 15.5 & 3.7 & 33.5 & - & 9.2 & & - & & - & - \\
\hline 8 & $129.5-139.0$ & 133.1 & 84.3 & - & 18.2 & 15.0 & 19.6 & 3.2 & 26.6 & - & 15.8 & & - & & 1.5 & - \\
\hline 9 & $139.0-148.5$ & 142.5 & 76.2 & 29.5 & 18.1 & 9.8 & 11.5 & - & 23.1 & 1.7 & 5.0 & & - & & 1.3 & - \\
\hline \multirow[t]{2}{*}{10} & $148.5-158.0$ & 153.8 & 16.2 & 94.1 & 2.3 & - & 1.2 & - & 2.5 & - & - & & - & & - & - \\
\hline & & 155.6 & 61.7 & 47.6 & 13.4 & 6.8 & 8.2 & - & 14.5 & 2.1 & 2.2 & & 5.2 & & - & - \\
\hline 15 & $234.0-243.5$ & 237.9 & 49.0 & 52.8 & 16.4 & 4.7 & 8.3 & - & 14.8 & 1.5 & 1.4 & & - & & - & - \\
\hline 16 & $243.5-253.0$ & 246.2 & 64.1 & 20.3 & 29.1 & 7.8 & 12.0 & - & 26.6 & 2.1 & 2.1 & & - & & - & - \\
\hline 17 & $253.0-262.5$ & 259.9 & 9.4 & 96.7 & 1.9 & - & - & - & 1.4 & - & - & & - & & - & - \\
\hline 21 & $329.0-338.5$ & 333.1 & 46.6 & 62.8 & 13.2 & 2.9 & 5.7 & - & 13.6 & 0.8 & 1.0 & & - & & - & - \\
\hline \multicolumn{17}{|c|}{ 2-20 $\mu \mathrm{m}$ Fractions } \\
\hline 2 & $25.0-34.5$ & 29.0 & 95.0 & & 18.4 & 24.5 & 32.9 & - & 5.0 & - & - & & & - & - & 19.2 \\
\hline 5 & $82.0-91.5$ & 89.0 & 77.5 & & 26.3 & 19.9 & 30.2 & 2.3 & 11.4 & - & - & & & - & 2.1 & 7.8 \\
\hline 6 & $101.0-110.5$ & 106.2 & 92.6 & & 38.2 & 17.9 & 27.7 & 5.3 & 10.9 & - & - & & & - & - & - \\
\hline 8 & $129.5-139.0$ & 133.1 & 83.7 & & 28.0 & 21.3 & 29.8 & 2.2 & 11.1 & - & 4.8 & & & - & 2.7 & - \\
\hline 9 & $139.0-148.5$ & 142.5 & 80.8 & & 34.8 & 20.2 & 25.1 & - & 15.4 & 2.0 & - & & & - & 2.5 & - \\
\hline \multirow[t]{2}{*}{10} & $148.5-158.0$ & 153.8 & 82.9 & & 38.8 & 12.2 & 22.6 & - & 19.8 & 4.7 & - & & & - & 1.9 & - \\
\hline & & 155.6 & 77.5 & & 39.6 & 12.1 & 26.0 & - & 17.7 & 2.6 & - & & & - & 2.1 & - \\
\hline 15 & $234.0-243.5$ & 237.9 & 51.5 & & 44.7 & 14.2 & 17.2 & - & 17.9 & 2.0 & - & & & 2.3 & 1.8 & - \\
\hline 16 & $243.5-253.0$ & 246.2 & 56.4 & & 46.1 & 12.5 & 17.4 & - & 16.3 & 2.2 & - & & & 4.2 & 1.2 & - \\
\hline 17 & $253.0-262.5$ & 259.9 & 71.7 & & 51.9 & 13.5 & 19.7 & - & 10.8 & 1.3 & - & & & 1.9 & 1.1 & - \\
\hline 21 & $329.0-338.5$ & 333.1 & 57.9 & & 46.2 & 15.5 & 17.1 & 0.4 & 17.3 & 1.4 & - & & & - & 2.2 & - \\
\hline \multicolumn{17}{|c|}{$<2 \mu \mathrm{m}$ Fractions } \\
\hline 2 & $25.0-34.5$ & 29.0 & 89.0 & & 11.5 & 18.7 & 19.6 & 4.3 & 11.4 & - & 27.1 & - & & & - & 7.6 \\
\hline 5 & $82.0-91.5$ & 89.0 & 80.7 & & 18.3 & 14.3 & 14.5 & 7.0 & 20.6 & - & 25.3 & - & & & - & - \\
\hline 6 & $101.0-110.5$ & 106.2 & 87.1 & & 19.7 & 12.7 & 19.3 & 3.3 & 23.1 & - & 21.9 & - & & & - & - \\
\hline 8 & $129.5-139.0$ & 133.1 & 86.4 & & 22.8 & 13.6 & 14.8 & 4.1 & 20.9 & - & 23.8 & - & & & - & - \\
\hline 9 & $139.0-148.5$ & 142.5 & 82.6 & & 26.0 & 13.6 & 19.9 & - & 24.0 & 3.7 & 12.9 & - & & & - & - \\
\hline \multirow[t]{2}{*}{10} & $148.5-158.0$ & 153.8 & 76.2 & & 31.8 & 7.9 & 16.5 & - & 28.7 & 4.6 & 10.5 & - & & & - & - \\
\hline & & 155.6 & 75.0 & & 28.0 & 6.2 & 17.0 & - & 24.2 & 3.4 & 9.0 & 12.3 & & & - & - \\
\hline 15 & $234.0-243.5$ & 237.9 & 69.7 & & 31.6 & 11.1 & 17.8 & - & 25.0 & 3.7 & 9.7 & - & & & 1.0 & - \\
\hline 16 & $243.5-253.0$ & 246.2 & 72.3 & & 33.4 & 10.2 & 16.0 & - & 28.2 & 3.9 & 8.2 & - & & & - & - \\
\hline 17 & $253.0-262.5$ & 259.9 & 76.3 & & 31.6 & 8.8 & 15.3 & - & 32.5 & 4.5 & 7.4 & - & & & - & - \\
\hline 21 & $329.0-338.5$ & 333.1 & 69.5 & & 31.6 & 11.0 & 17.9 & - & 25.8 & 2.3 & 10.1 & - & & & 1.4 & - \\
\hline
\end{tabular}

TABLE 15

Results of X-Ray Diffraction Analysis From Hole 267

\begin{tabular}{|c|c|c|c|c|c|c|c|c|c|c|c|}
\hline Core & $\begin{array}{c}\text { Cored } \\
\text { Interval } \\
\text { Below Sea } \\
\text { Floor (m) }\end{array}$ & $\begin{array}{l}\text { Sample Depth } \\
\text { Below Sea } \\
\text { Floor }(\mathrm{m})\end{array}$ & 客 & లే & 䒕 & $\frac{\dot{0}}{\dot{1}}$ & ङ & 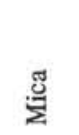 & ही & 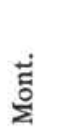 & 容 \\
\hline \multicolumn{12}{|c|}{ Bulk Samples } \\
\hline 3 & $89.5-99.0$ & 94.8 & 59.8 & - & 30.8 & 8.9 & 17.1 & 32.9 & 2.1 & 7.3 & 0.9 \\
\hline 4 & $127.5-137.0$ & 132.8 & 37.5 & 68.1 & 12.4 & 3.2 & 5.3 & 10.3 & 0.8 & - & - \\
\hline \multicolumn{12}{|c|}{$2-20 \mu \mathrm{m}$ Fractions } \\
\hline 3 & $89.5-99.0$ & 94.8 & 39.5 & & 42.4 & 12.7 & 20.7 & 20.7 & 1.8 & & 1.6 \\
\hline 4 & $127.5-137.0$ & 132.8 & 48.2 & & 47.2 & 9.7 & 17.2 & 21.9 & 1.9 & & 2.0 \\
\hline \multicolumn{12}{|c|}{$<2 \mu \mathrm{m}$ Fractions } \\
\hline 3 & $89.5-99.0$ & 94.8 & 62.7 & & 30.9 & 10.9 & 17.6 & 30.6 & 2.5 & 7.4 & \\
\hline 4 & $127.5-137.0$ & 132.8 & 65.3 & & 36.5 & 9.9 & 18.4 & 26.7 & 3.2 & 5.2 & \\
\hline
\end{tabular}


TABLE 16

Results of X-Ray Diffraction Analysis From Hole 267A

\begin{tabular}{|c|c|c|c|c|c|c|c|c|c|c|c|}
\hline Core & $\begin{array}{c}\text { Cored } \\
\text { Interval } \\
\text { Below Sea } \\
\text { Floor (m) }\end{array}$ & $\begin{array}{l}\text { Sample Depth } \\
\text { Below Sea } \\
\text { Floor }(\mathrm{m})\end{array}$ & 范 & 岁 & 选 & $\begin{array}{l}\stackrel{00}{0} \\
\frac{\pi}{2}\end{array}$ & ठ্ّ & 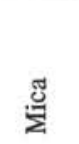 & 己ี & 范 & 完 \\
\hline \multicolumn{12}{|c|}{ Bulk Samples } \\
\hline 1 & $4.0-13.5$ & 11.8 & 76.4 & 29.5 & 14.8 & 16.5 & - & 32.3 & 0.5 & 4.3 & 2.2 \\
\hline 3 & $61.0-70.5$ & 63.5 & 59.4 & 37.6 & - & 17.1 & 3.3 & 42.0 & - & - & - \\
\hline \multicolumn{12}{|c|}{$2-20 \mu \mathrm{m}$ Fractions } \\
\hline 1 & $4.0-13.5$ & 11.8 & 73.3 & 45.8 & 18.6 & 25.2 & & 10.5 & - & & - \\
\hline 3 & $61.0-70.5$ & 63.5 & 45.1 & 41.3 & 12.8 & 19.5 & & 22.8 & 1.6 & & 2.0 \\
\hline \multicolumn{12}{|c|}{$<2 \mu \mathrm{m}$ Fractions } \\
\hline 1 & $4.0-13.5$ & 11.8 & 81.4 & 27.6 & 17.8 & 16.6 & - & 30.7 & 1.3 & 6.1 & \\
\hline 3 & $61.0-70.5$ & 63.5 & 68.8 & 29.6 & 11.3 & 15.9 & 1.8 & 29.2 & 1.1 & 11.0 & \\
\hline
\end{tabular}

TABLE 17

Results of X-Ray Diffraction Analysis From Hole 267B

\begin{tabular}{|c|c|c|c|c|c|c|c|c|c|c|c|c|c|}
\hline Core & $\begin{array}{c}\text { Cored } \\
\text { Interval } \\
\text { Below Sea } \\
\text { Floor }(\mathrm{m})\end{array}$ & $\begin{array}{c}\text { Sample Depth } \\
\text { Below Sea } \\
\text { Floor (m) }\end{array}$ & 安 & $\begin{array}{l}\text { ठ் } \\
\text { ‡ }\end{array}$ & 芫 & 离 & $\frac{a}{\pi}$ & 总 & $\stackrel{\mathscr{g}}{\Sigma}$ & ฮี่ & $\frac{\dot{E}}{\dot{\Sigma}}$ & $\dot{\Xi}$ & 总 \\
\hline \multicolumn{14}{|c|}{ Bulk Samples } \\
\hline 1 & $105.0-114.5$ & 106.1 & 71.1 & - & 22.8 & 15.2 & 18.1 & 1.2 & 34.5 & 0.6 & 5.4 & - & 2.2 \\
\hline 3 & $143.0-152.5$ & 146.0 & 71.3 & - & 26.5 & 12.2 & 17.1 & 2.1 & 32.4 & 1.5 & 6.6 & - & 1.6 \\
\hline 4 & $162.0-171.5$ & 164.5 & 70.5 & 12.1 & 23.6 & 9.3 & 12.5 & 1.5 & 33.2 & 2.1 & 1.1 & 3.6 & 1.0 \\
\hline 6 & $209.5-219.0$ & 217.4 & 66.9 & - & 29.7 & 9.8 & 16.1 & 2.7 & 33.1 & 2.2 & 5.3 & - & 1.0 \\
\hline 7 & $238.0-147.5$ & 246.1 & 63.0 & - & 31.1 & 10.6 & 16.6 & 1.4 & 33.5 & 1.2 & 5.5 & - & - \\
\hline 8 & $266.5-276.0$ & 273.3 & 59.5 & - & 33.1 & 10.8 & 16.9 & - & 31.8 & 1.6 & 4.7 & - & 1.0 \\
\hline 9 & $295.0-304.5$ & 302.1 & 58.2 & - & 34.5 & 11.4 & 17.1 & - & 30.4 & 1.6 & 3.7 & - & 1.4 \\
\hline \multicolumn{14}{|c|}{$2-20 \mu \mathrm{m}$ Fractions } \\
\hline 1 & $105.0-114.5$ & 106.1 & 60.9 & & 35.6 & 22.9 & 27.0 & & 11.0 & - & & & 3.5 \\
\hline 3 & $143.0-152.5$ & 146.0 & 61.0 & & 36.8 & 16.2 & 20.2 & & 21.5 & 3.4 & & & 1.9 \\
\hline 4 & $162.0-171.5$ & 164.5 & 64.5 & & 39.5 & 15.6 & 19.6 & & 21.1 & 2.3 & & & 1.8 \\
\hline 6 & $209.5-219.0$ & 217.4 & 51.5 & & 41.0 & 12.9 & 20.1 & & 22.2 & 1.7 & & & 2.2 \\
\hline 7 & $238.0-247.5$ & 246.1 & 48.4 & & 42.3 & 12.9 & 19.4 & & 22.6 & 0.8 & & & 1.9 \\
\hline 8 & $266.5-276.0$ & 273.3 & 36.3 & & 48.5 & 14.9 & 18.5 & & 15.0 & 0.9 & & & 2.2 \\
\hline 9 & $295.0-304.5$ & 302.1 & 29.9 & & 39.3 & 11.3 & 18.3 & & 27.2 & 1.6 & & & 2.3 \\
\hline \multicolumn{14}{|c|}{$<2 \mu \mathrm{m}$ Fractions } \\
\hline 1 & $105.0-114.5$ & 106.1 & 78.6 & & 21.9 & 14.2 & 21.0 & 2.7 & 19.0 & 0.6 & 8.3 & & 2.2 \\
\hline 3 & $143.0-152.5$ & 146.0 & 76.1 & & 24.8 & 13.3 & 17.8 & 4.7 & 24.3 & 1.7 & 13.3 & & - \\
\hline 4 & $162.0-171.5$ & 164.5 & 74.2 & & 24.3 & 10.3 & 15.4 & 4.6 & 31.0 & 2.3 & 12.3 & & - \\
\hline 6 & $209.5-219.0$ & 217.4 & 71.7 & & 29.9 & 9.3 & 16.1 & 2.4 & 32.3 & 2.1 & 7.9 & & - \\
\hline 7 & $238.0-247.5$ & 246.1 & 67.8 & & 26.0 & 10.9 & 15.2 & 4.4 & 26.0 & - & 17.6 & & - \\
\hline 8 & $266.5-276.0$ & 273.3 & 64.4 & & 29.2 & 10.0 & 16.7 & 1.5 & 30.6 & 1.2 & 10.9 & & - \\
\hline 9 & $295.0-304.5$ & 302.1 & 62.5 & & 30.5 & 11.4 & 18.6 & 1.0 & 29.8 & 2.1 & 6.7 & & - \\
\hline
\end{tabular}


TABLE 18

Results of X-Ray Diffraction Analy sis From Site 268

\begin{tabular}{|c|c|c|c|c|c|c|c|c|c|c|c|c|c|c|c|c|}
\hline Core & $\begin{array}{c}\text { Cored } \\
\text { Interval } \\
\text { Below Sea } \\
\text { Floor }(\mathrm{m})\end{array}$ & $\begin{array}{l}\text { Sample Depth } \\
\text { Below Sea } \\
\text { Floor }(\mathrm{m})\end{array}$ & 岁 & गี่ & పేँ & $\dot{\ddot{Z}}$ & 㭊 & $\stackrel{g}{a}$ & $\begin{array}{l}\dot{0} \\
\ddot{\varpi}\end{array}$ & $\stackrel{\circlearrowright}{\stackrel{0}{\Sigma}}$ & हो & 范 & 总 & 惡 & 完 & 咅 \\
\hline \multicolumn{17}{|c|}{ Bulk Samples } \\
\hline \multirow[t]{2}{*}{2} & $28.0-37.5$ & 33.1 & 42.1 & - & 36.8 & - & 19.8 & 17.5 & 1.3 & 21.3 & 2.1 & - & & - & - & 1.1 \\
\hline & & 33.2 & 53.7 & - & 29.7 & - & 16.7 & 18.8 & 1.5 & 26.9 & 1.7 & 2.7 & & - & - & 2.0 \\
\hline \multirow[t]{2}{*}{8} & $170.5-180.0$ & 170.9 & 47.8 & - & 44.4 & - & 12.6 & 14.1 & - & 24.7 & 3.0 & - & & - & - & 1.2 \\
\hline & & 171.2 & 47.9 & - & 36.2 & - & 9.8 & 12.6 & - & 33.6 & 3.9 & - & & 2.7 & - & 1.2 \\
\hline 10 & $227.5-237.0$ & 229.4 & 48.3 & - & 40.4 & - & 16.7 & 15.1 & - & 22.4 & 2.6 & - & & 1.7 & - & 1.0 \\
\hline \multirow[t]{3}{*}{13} & $303.5-313.0$ & 304.3 & 48.9 & - & 21.1 & 48.7 & 6.8 & 9.6 & - & 10.6 & 1.6 & - & & 1.6 & - & - \\
\hline & & 304.3 & 50.2 & 6.1 & 26.6 & - & 10.1 & 17.3 & - & 30.7 & 5.0 & 4.2 & & - & - & - \\
\hline & & 304.5 & 22.8 & - & 48.6 & 3.6 & 15.8 & 15.4 & - & 13.6 & 1.8 & - & & - & - & 1.2 \\
\hline 17 & $379.5-389.0$ & 380.9 & 33.6 & - & 50.5 & - & 15.4 & 12.4 & - & 18.6 & 2.0 & - & & - & - & 1.1 \\
\hline 20 & $465.0-474.5$ & 467.3 & 41.2 & - & 45.8 & - & 14.9 & 10.8 & - & 17.4 & 2.4 & - & & - & 8.7 & - \\
\hline \multicolumn{17}{|c|}{$2-20 \mu \mathrm{m}$ Fractions } \\
\hline \multirow[t]{2}{*}{2} & $28.0-37.5$ & 33.1 & 11.8 & & 45.9 & & 19.6 & 18.4 & & 12.0 & 0.5 & - & - & - & - & 3.6 \\
\hline & & 33.2 & 32.5 & & 39.9 & & 17.9 & 21.4 & & 15.6 & 1.3 & - & - & - & - & 3.8 \\
\hline \multirow[t]{2}{*}{8} & $170.4-180.0$ & 170.9 & 19.0 & & 55.2 & & 15.1 & 17.5 & & 8.8 & 1.4 & - & - & - & - & 2.0 \\
\hline & & 171.2 & 13.4 & & 55.4 & & 9.5 & 16.5 & & 12.2 & 2.4 & - & - & 2.1 & - & 1.9 \\
\hline 10 & $227.5-237.0$ & 229.4 & 17.2 & & 49.9 & & 13.3 & 17.3 & & 11.3 & 1.5 & - & 5.4 & 1.2 & - & - \\
\hline \multirow[t]{2}{*}{13} & $303.5-313.0$ & 304.3 & 22.1 & & 42.2 & & 9.9 & 18.3 & & 22.3 & 5.3 & 0.9 & - & - & - & 1.1 \\
\hline & & 304.5 & - & & 56.8 & & 13.9 & 17.5 & & 8.1 & 1.2 & - & - & - & - & 2.5 \\
\hline 17 & $379.5-389.0$ & 380.9 & 2.2 & & 57.7 & & 15.2 & 12.7 & & 11.0 & 1.7 & - & - & - & - & 1.7 \\
\hline 20 & $465.0-474.5$ & 467.3 & 15.8 & & 53.1 & & 13.7 & 13.1 & & 11.1 & 1.7 & - & - & - & 7.4 & - \\
\hline \multicolumn{17}{|c|}{$<2 \mu \mathrm{m}$ Fractions } \\
\hline \multirow[t]{2}{*}{2} & $28.0-37.5$ & 33.1 & 59.2 & & 27.7 & - & 16.5 & 15.2 & 5.7 & 30.5 & 1.7 & 2.7 & & - & - & - \\
\hline & & 33.2 & 70.5 & & 24.3 & - & 11.9 & 17.1 & 4.5 & 31.8 & 2.9 & 6.2 & & - & - & 1.4 \\
\hline \multirow[t]{2}{*}{8} & $170.5-180.0$ & 170.9 & 55.8 & & 33.4 & - & 9.4 & 13.0 & - & 36.5 & 6.7 & - & & - & - & 1.0 \\
\hline & & 171.2 & 56.4 & & 30.9 & - & 10.5 & 15.2 & - & 34.6 & 4.7 & 1.2 & & 3.0 & - & - \\
\hline 10 & $227.5-237.0$ & 229.4 & 65.3 & & 29.7 & - & 11.3 & 15.7 & - & 33.2 & 5.0 & 3.4 & & 1.6 & - & - \\
\hline \multirow[t]{2}{*}{13} & $303.5-313.0$ & 304.3 & 59.1 & & 26.6 & - & 8.7 & 21.7 & - & 28.6 & 6.0 & 8.6 & & - & - & - \\
\hline & & 304.5 & 55.1 & & 27.2 & 7.7 & 12.0 & 14.8 & - & 30.9 & 4.3 & 3.1 & & - & - & - \\
\hline 17 & $379.5-389.0$ & 380.9 & 54.2 & & 40.3 & - & 14.3 & 12.8 & - & 27.5 & 5.1 & - & & - & - & - \\
\hline 20 & $465.0-474.5$ & 467.3 & 64.2 & & 33.7 & - & 13.2 & 11.9 & - & 30.4 & 4.5 & 2.1 & & - & 4.2 & - \\
\hline
\end{tabular}


TABLE 19

Results of X-Ray Diffraction Analysis From Hole 269

\begin{tabular}{|c|c|c|c|c|c|c|c|c|c|c|c|c|c|}
\hline Core & $\begin{array}{c}\text { Cored } \\
\text { Interval } \\
\text { Below Sea } \\
\text { Floor (m) }\end{array}$ & $\begin{array}{l}\text { Sample Depth } \\
\text { Below Sea } \\
\text { Floor }(\mathrm{m})\end{array}$ & 冚 & है & 峷 & घี & वं & $\stackrel{\Xi}{\frac{0}{2}}$ & 己̇ं & हूँ & 岂 & 完 & $\stackrel{\text { ָే }}{3}$ \\
\hline \multicolumn{14}{|c|}{ Bulk Samples } \\
\hline 2 & $46.0-55.5$ & 47.6 & 49.5 & 23.0 & 3.2 & 9.7 & & 52.9 & 4.4 & 6.7 & - & - & \\
\hline 3 & $93.5-103.0$ & 95.6 & 54.1 & 35.2 & 8.7 & 15.8 & & 33.8 & 3.6 & 2.9 & - & - & \\
\hline 6 & 198.0-207.5 & 203.3 & 52.8 & 32.6 & 11.9 & 18.4 & & 31.6 & 4.5 & - & - & 1.0 & \\
\hline 9 & $331.0-340.5$ & 331.7 & 45.1 & 45.5 & 15.8 & 23.2 & & 10.6 & 1.9 & 1.8 & 1.3 & - & \\
\hline & & 333.2 & 59.8 & 35.1 & 13.5 & 21.2 & & 21.9 & 4.3 & 4.1 & - & - & \\
\hline 11 & $388.0-397.5$ & 389.6 & 53.1 & 35.1 & 7.1 & 20.7 & & 27.8 & 4.6 & 3.8 & - & 0.9 & \\
\hline \multicolumn{14}{|c|}{ 2-20 $\mu \mathrm{m}$ Fractions } \\
\hline 2 & $46.0-55.5$ & 47.6 & 16.2 & 32.1 & 4.6 & 14.9 & & 42.3 & 6.2 & & - & - & $\mathrm{T}$ \\
\hline 3 & $93.5-103.0$ & 95.6 & 32.3 & 40.2 & 8.4 & 18.5 & & 28.3 & 4.6 & & - & - & $\mathrm{T}$ \\
\hline 6 & $198.0-207.5$ & 203.3 & 38.2 & 38.9 & 12.4 & 20.9 & & 22.7 & 3.8 & & - & 1.4 & $\mathrm{~T}$ \\
\hline 9 & $331.0-340.5$ & 331.7 & 28.2 & 46.6 & 16.6 & 22.8 & & 9.0 & 1.9 & & 1.2 & 2.0 & - \\
\hline & & 333.2 & 48.1 & 43.7 & 12.9 & 21.2 & & 17.8 & 2.7 & & - & 1.7 & $\mathrm{~T}$ \\
\hline 11 & $388.0-397.5$ & 389.6 & 27.8 & 46.6 & 11.5 & 21.6 & & 16.2 & 2.3 & & - & 1.8 & $\mathrm{~T}$ \\
\hline \multicolumn{14}{|c|}{$<2 \mu \mathrm{m}$ Fractions } \\
\hline 2 & $46.0-55.5$ & 47.6 & 65.1 & 26.2 & 5.9 & 10.0 & 4.1 & 35.8 & 3.1 & 14.8 & & & \\
\hline 3 & $93.5-103.0$ & 95.6 & 69.9 & 28.1 & 7.4 & 15.2 & - & 32.7 & 5.3 & 11.3 & & & \\
\hline 6 & $198.0-207.5$ & 203.3 & 73.3 & 29.6 & 14.1 & 19.1 & - & 24.2 & 5.9 & 7.1 & & & \\
\hline 9 & $331.0-340.5$ & 331.7 & 68.8 & 31.1 & 12.8 & 22.7 & - & 17.3 & 4.6 & 11.5 & & & \\
\hline & & 333.2 & 66.2 & 32.7 & 15.5 & 23.5 & - & 16.5 & 4.5 & 7.4 & & & \\
\hline 11 & $388.0-397.5$ & 389.6 & 67.4 & 36.4 & 10.0 & 24.5 & - & 15.8 & 3.6 & 9.8 & & & \\
\hline
\end{tabular}

${ }^{\mathrm{a}} \mathrm{U}-2$ peak at $12.1 \AA$. 
TABLE 20

Results of X-Ray Diffraction Analysis From Hole 269A

\begin{tabular}{|c|c|c|c|c|c|c|c|c|c|c|c|c|c|c|c|c|}
\hline Core & $\begin{array}{c}\text { Cored } \\
\text { Interval } \\
\text { Below Sea } \\
\text { Floor }(\mathrm{m})\end{array}$ & $\begin{array}{l}\text { Sample Depth } \\
\text { Below Sea } \\
\text { Floor }(\mathrm{m})\end{array}$ & 客 & 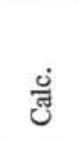 & हैं & $\dot{\ddot{H}}$ & 这 & $\frac{\tilde{m}}{\tilde{a}}$ & 元 & ठํ & $\stackrel{+}{\text { 듈 }}$ & 总 & छ் & 完 & స్ ָే & 今. \\
\hline \multicolumn{17}{|c|}{ Bulk Samples } \\
\hline 2 & $426.0-435.5$ & 428.4 & 52.7 & 10.4 & 35.7 & - & 13.7 & 19.4 & 16.4 & 2.2 & 1.2 & - & & 1.1 & & \\
\hline \multirow[t]{2}{*}{3} & $473.5-483.0$ & 475.2 & 51.9 & - & 28.1 & 29.3 & 7.0 & 13.5 & 15.3 & 1.9 & 4.9 & - & & - & & \\
\hline & & 476.1 & 51.5 & - & 32.2 & 11.9 & 10.6 & 15.8 & 19.6 & 2.6 & 5.2 & 2.1 & & - & & \\
\hline \multirow[t]{2}{*}{4} & $521.0-530.5$ & 523.0 & 33.8 & - & 29.9 & 23.3 & 8.5 & 15.3 & 15.3 & 2.1 & 1.6 & 3.0 & & 0.9 & & \\
\hline & & 523.5 & 52.5 & - & 35.4 & 10.5 & 11.0 & 16.4 & 17.3 & 3.0 & 6.3 & - & & - & & \\
\hline \multirow[t]{3}{*}{8} & $701.5-711.0$ & 702.5 & 32.9 & - & 38.6 & - & 7.9 & 13.1 & 16.8 & 3.2 & 20.3 & - & & - & & \\
\hline & & 703.5 & 28.2 & - & 43.9 & - & 11.8 & 18.6 & 18.1 & 2.4 & 5.2 & - & & - & & \\
\hline & & 703.6 & 23.5 & - & 38.8 & - & 8.7 & 16.4 & 16.0 & 2.2 & 18.0 & - & & - & & \\
\hline 10 & $806.0-815.5$ & 808.3 & 33.0 & - & 45.7 & - & 12.0 & 18.6 & 13.8 & 2.3 & 7.6 & - & & - & & \\
\hline 12 & $901.0-910.5$ & 907.0 & 39.5 & - & 42.4 & - & 5.8 & 16.1 & 17.2 & 3.0 & 15.5 & - & & - & & \\
\hline 13 & $948.5-958.0$ & 954.3 & 33.2 & - & 29.2 & - & 5.3 & 17.2 & 36.8 & 6.8 & 4.7 & - & & - & & \\
\hline \multicolumn{17}{|c|}{$2-20 \mu \mathrm{m}$ Fractions } \\
\hline 2 & $426.0-435.5$ & 428.4 & 33.1 & & 40.9 & - & 15.3 & 21.7 & 18.1 & 2.7 & - & - & - & 1.3 & P & - \\
\hline 3 & $473.5-483.0$ & 475.2 & 32.9 & & 41.5 & 13.8 & 9.3 & 17.5 & 14.3 & 1.9 & - & 0.8 & - & 0.8 & - & - \\
\hline \multirow[t]{2}{*}{4} & $521.0-530.5$ & 523.0 & 19.9 & & 38.0 & 10.8 & 10.2 & 18.8 & 17.0 & 2.5 & - & 1.4 & - & 1.3 & $\mathrm{~T}$ & - \\
\hline & & 523.5 & 28.0 & & 48.6 & - & 10.2 & 18.2 & 19.6 & 3.4 & - & - & - & - & $P$ & - \\
\hline \multirow[t]{3}{*}{8} & $701.5-711.0$ & 702.5 & 7.5 & & 36.2 & - & 6.4 & 13.0 & 13.0 & 2.8 & 28.7 & - & - & - & $\mathrm{T}$ & - \\
\hline & & 703.5 & 2.8 & & 51.5 & - & 11.3 & 19.2 & 14.5 & 2.5 & - & - & - & 1.2 & $\mathrm{~T}$ & - \\
\hline & & 703.6 & 4.1 & & 43.8 & - & 10.5 & 17.0 & 17.2 & 3.1 & 8.4 & - & - & - & $\mathrm{T}$ & $\mathrm{T}$ \\
\hline 10 & $806.0-815.5$ & 808.3 & 4.8 & & 50.1 & - & 11.5 & 17.2 & 12.7 & 1.9 & 5.7 & - & - & 0.9 & - & $\mathbf{P}$ \\
\hline 12 & $901.0-910.5$ & 907.0 & 7.0 & & 34.5 & - & 5.8 & 13.4 & 17.0 & 3.1 & 25.4 & - & 0.8 & - & $\mathrm{T}$ & - \\
\hline 13 & $948.5-958.0$ & 954.3 & 4.9 & & 29.1 & - & 5.1 & 15.9 & 31.4 & 7.1 & 11.3 & - & - & - & $\mathrm{T}$ & - \\
\hline \multicolumn{17}{|c|}{$<2 \mu \mathrm{m}$ Fractions } \\
\hline 2 & $426.0-435.5$ & 428.4 & 71.7 & & 28.9 & - & 12.4 & 22.0 & 17.8 & 3.1 & 15.8 & - & & & & \\
\hline 3 & $473.5-483.0$ & 475.2 & 59.3 & & 26.3 & 33.3 & 8.8 & 15.2 & 6.3 & 1.6 & 7.3 & 1.2 & & & & \\
\hline \multirow[t]{2}{*}{4} & $521.0-530.5$ & 523.0 & 51.2 & & 11.1 & 56.0 & 4.6 & 7.9 & 10.3 & 1.3 & 5.4 & 3.4 & & & & \\
\hline & & 523.5 & 61.3 & & 36.1 & 10.9 & 12.5 & 16.8 & 10.4 & 2.9 & 10.3 & - & & & & \\
\hline \multirow[t]{3}{*}{8} & $701.5-711.0$ & 702.5 & 50.0 & & 34.0 & - & 6.5 & 12.5 & 11.0 & 3.3 & 32.8 & - & & & & \\
\hline & & 703.5 & 50.4 & & 37.8 & - & 9.0 & 17.8 & 17.7 & 3.6 & 14.1 & - & & & & \\
\hline & & 703.6 & 51.7 & & 34.4 & - & 7.3 & 14.6 & 18.6 & 3.2 & 21.8 & - & & & & \\
\hline 10 & $806.0-815.5$ & 808.3 & 49.6 & & 34.7 & - & 10.6 & 19.4 & 16.2 & 3.1 & 15.9 & - & & & & \\
\hline 12 & $901.0-910.5$ & 907.0 & 46.0 & & 31.8 & - & 6.0 & 15.5 & 13.4 & 2.9 & 30.5 & - & & & & \\
\hline 13 & $948.5-958.0$ & 954.3 & 45.2 & & 18.4 & - & 4.0 & 16.2 & 29.3 & 5.3 & 26.8 & - & & & & \\
\hline
\end{tabular}

${ }^{\mathrm{a}} \mathrm{U}-2$ peak at $12.1 \AA$.

${ }^{\mathrm{b}} \mathrm{U}-3$ peaks at $22.2 \AA$ and $11.25 \AA$. 
TABLE 21

Results of X-Ray Diffraction Analysis From Site 270

\begin{tabular}{|c|c|c|c|c|c|c|c|c|c|c|c|c|c|c|c|c|c|c|c|c|}
\hline & Cored & & & & & & & & & & & * & & & & & & & , & \\
\hline Core & $\begin{array}{l}\text { Interval } \\
\text { Below Sea } \\
\text { Floor (m) }\end{array}$ & $\begin{array}{l}\text { Sample Depth } \\
\text { Below Sea } \\
\text { Floor }(\mathrm{m})\end{array}$ & 它 & 芦 & تृ & कृ & है & $\stackrel{\dot{U}}{\mathrm{C}}$ & $\frac{0}{2}$ & $\frac{a j}{a}$ & $\begin{array}{l}\dot{0} \\
\ddot{g}\end{array}$ & $\frac{\pi}{2}$ & $\frac{\circ}{E}$ & 侖 & 总 & 톨 & 忘 & 芯 & 音 & 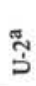 \\
\hline
\end{tabular}

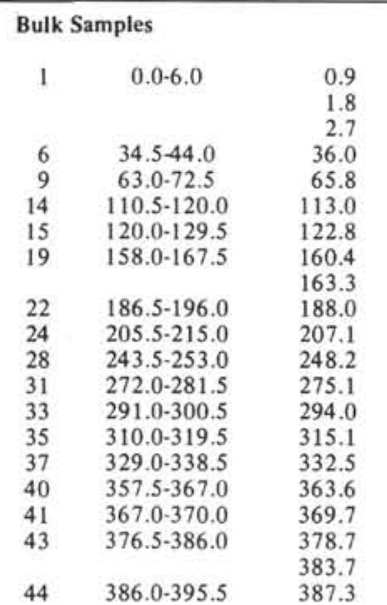

\begin{tabular}{|c|c|c|c|c|c|c|c|c|c|c|c|c|c|c|c|}
\hline 49.6 & - & - & - & 33.0 & - & 9.3 & 17.3 & - & 37.6 & 2.8 & - & - & - & - & - \\
\hline 33.2 & - & - & - & 35.8 & - & 14.3 & 21.3 & - & 24.4 & 1.9 & 2.4 & - & - & - & - \\
\hline 26.1 & - & - & - & 36.9 & - & 13.1 & 21.4 & - & 27.5 & 1.2 & - & - & - & - & - \\
\hline 29.7 & - & - & - & 25.7 & 28.4 & 11.8 & 18.8 & - & 10.8 & 1.3 & - & 2.4 & 0.7 & - & - \\
\hline 28.7 & - & - & - & 26.7 & 29.5 & 8.5 & 19.3 & - & 10.8 & 1.5 & - & 2.5 & 0.6 & - & 0.7 \\
\hline 35.4 & - & - & - & 28.7 & 15.1 & 7.9 & 23.3 & - & 18.2 & 3.3 & 0.8 & - & 0.6 & 2.1 & - \\
\hline 32.2 & - & - & - & 29.5 & 16.4 & 11.0 & 25.3 & - & 14.2 & 3.0 & - & - & 0.7 & - & - \\
\hline 29.6 & - & - & - & 31.4 & 11.9 & 9.2 & 23.4 & - & 19.4 & 3.6 & 1.2 & - & - & - & - \\
\hline 30.9 & - & - & - & 28.4 & 10.8 & 8.4 & 22.3 & - & 21.7 & 4.6 & 2.8 & - & 0.9 & - & - \\
\hline 35.4 & - & - & - & 29.9 & - & 15.1 & 36.2 & - & 12.7 & 3.6 & - & - & 1.4 & - & 1.2 \\
\hline 18.9 & - & - & - & 41.0 & - & 12.7 & 20.5 & 0.4 & 21.1 & 3.4 & - & - & 0.9 & - & - \\
\hline 20.2 & - & - & - & 43.0 & - & 14.9 & 22.8 & - & 16.6 & 2.7 & - & - & - & - & - \\
\hline 28.5 & - & - & - & 38.6 & - & 11.1 & 22.6 & - & 20.8 & 3.4 & 3.5 & - & - & - & - \\
\hline 26.5 & - & - & - & 39.4 & - & 13.6 & 21.8 & - & 19.1 & 3.1 & 2.9 & - & - & - & - \\
\hline 30.0 & - & - & - & 20.2 & - & 9.3 & 12.6 & - & 12.6 & 20.0 & 25.3 & - & - & - & - \\
\hline 28.4 & - & - & - & 33.7 & - & 7.6 & 19.6 & - & 30.2 & 5.2 & 3.6 & - & - & - & - \\
\hline 19.9 & - & - & - & 41.2 & - & 14.4 & 18.8 & - & 19.4 & 3.7 & 2.5 & - & - & - & - \\
\hline 25.8 & - & - & - & 41.4 & - & 9.9 & 16.1 & 1.3 & 23.5 & 3.6 & 4.2 & - & - & - & - \\
\hline 12.8 & 8.0 & 3.2 & 2.2 & 48.7 & - & 16.6 & 13.9 & 3.1 & 4.3 & - & - & - & - & - & - \\
\hline 26.8 & - & - & 3.5 & 42.5 & - & 8.7 & 7.4 & 11.3 & 23.2 & - & 2.2 & - & 1.2 & - & - \\
\hline 9.4 & - & - & - & 46.9 & - & 26.8 & - & 6.4 & 7.1 & - & 10.6 & - & 2.1 & - & - \\
\hline
\end{tabular}

2-20 $\mu \mathrm{m}$ Fractions

$\begin{array}{rcr}1 & 0.0-6.0 & 0.9 \\ & & 1.8 \\ & & 2.7 \\ 6 & 34.5-44.0 & 36.0 \\ 9 & 63.0-72.5 & 65.8 \\ 14 & 110.5-120.0 & 113.0 \\ 15 & 120.0-129.5 & 122.8 \\ 19 & 158.0-167.5 & 160.4 \\ & & 163.3 \\ 22 & 186.5-196.0 & 188.0 \\ 24 & 205.5-215.0 & 207.1 \\ 28 & 243.5-253.0 & 248.2 \\ 31 & 272.0-281.5 & 275.1 \\ 33 & 291.0-300.5 & 294.0 \\ 35 & 310.0-319.5 & 315.1 \\ 37 & 329.0-338.5 & 332.5 \\ 40 & 357.5-367.0 & 363.6 \\ 41 & 367.0-370.0 & 369.7 \\ 43 & 376.5-386.0 & 378.7 \\ & & 383.7 \\ 44 & 386.0-395.5 & 387.3\end{array}$

33.6
22.1
16.8
18.9
15.9
15.0
20.0
9.7
6.1
13.2
3.5
7.8
6.8
2.9
8.2
5.2
-
1.4
6.1
-
8.2

$\begin{array}{lllr}- & - & 40.8 & - \\ - & - & 41.5 & - \\ - & - & 37.3 & - \\ - & - & 24.0 & 27.9 \\ - & - & 29.1 & 20.3 \\ - & - & 30.4 & 9 . \\ - & - & 35.9 & - \\ - & - & 32.2 & 5.4 \\ - & - & 29.9 & 3.6 \\ - & - & 45.6 & - \\ - & - & 45.5 & - \\ - & - & 44.4 & - \\ - & - & 45.5 & - \\ - & - & 43.0 & - \\ - & - & 43.6 & - \\ - & - & 45.5 & - \\ 1.0 & 2.7 & 39.8 & - \\ - & 1.3 & 46.3 & - \\ - & - & 12.8 & -\end{array}$

\begin{tabular}{|c|c|}
\hline 8.6 & 25.5 \\
\hline 12.1 & 26.1 \\
\hline 9.1 & 22.4 \\
\hline 6.5 & 19.6 \\
\hline 7.2 & 19.9 \\
\hline 8.5 & 22.5 \\
\hline 9.6 & 26.0 \\
\hline 15.8 & 21.2 \\
\hline 9.3 & 23.3 \\
\hline 11.1 & 37.2 \\
\hline 8.1 & 21.1 \\
\hline 9.4 & 22.5 \\
\hline 7.8 & 24.5 \\
\hline 9.0 & 22.3 \\
\hline 7.6 & 24.7 \\
\hline 8.0 & 21.5 \\
\hline 8.7 & 18.3 \\
\hline 8.8 & 16.9 \\
\hline 8.6 & 15.4 \\
\hline 8.5 & 10.6 \\
\hline 15.5 & 1. \\
\hline
\end{tabular}

$\begin{array}{rrrll}- & 20.4 & 3.4 & - & - \\ - & 16.8 & 2.3 & - & - \\ - & 26.3 & 3.4 & - & - \\ - & 16.7 & 2.2 & - & 1.7 \\ - & 17.4 & 2.7 & - & 1.5 \\ - & 22.8 & 3.9 & - & 0.9 \\ - & 23.3 & 4.4 & - & - \\ - & 19.7 & 4.0 & - & - \\ - & 26.4 & 5.2 & - & - \\ - & 9.7 & 2.3 & - & - \\ - & 19.0 & 4.1 & - & - \\ - & 18.1 & 3.5 & - & - \\ - & 18.5 & 3.6 & - & - \\ - & 19.2 & 4.0 & - & - \\ - & 19.7 & 3.9 & - & - \\ - & 24.7 & 4.1 & - & - \\ - & 24.5 & 4.3 & - & - \\ 1.9 & 22.1 & 4.0 & - & - \\ 4.3 & 14.9 & 1.0 & - & - \\ 2.5 & 18.7 & - & - & - \\ 7.1 & 28.9 & - & 8.7 & -\end{array}$

$\begin{array}{lll}- & 1.3 & - \\ - & 1.3 & \overline{-} \\ 1.3 & 1.4 & \mathrm{~T} \\ 0.8 & -1.2 & - \\ 0.6 & 1.2 & - \\ 0.8 & - & - \\ 0.8 & 1.0 & - \\ 1.3 & 1.1 & - \\ 1.9 & 2.1 & - \\ 1.4 & - & - \\ 1.0 & - & - \\ 1.1 & - & - \\ - & - & - \\ 1.0 & - & - \\ - & - & - \\ 0.8 & - & - \\ 0.9 & - & - \\ 2.4 & - & - \\ 2.1 & - & - \\ 5.9 & - & -\end{array}$

$<2 \mu \mathrm{m}$ Fractions

\begin{tabular}{|c|c|c|c|c|c|c|c|c|c|c|c|c|c|c|c|}
\hline \multirow[t]{3}{*}{1} & $0.0-6.0$ & 0.9 & 73.7 & 17.7 & - & 9.2 & 15.6 & - & 44.0 & 6.5 & 6.9 & - & - & - & - \\
\hline & & 1.8 & 62.9 & 16.8 & - & 6.1 & 16.2 & - & 42.1 & 6.8 & 12.0 & - & - & - & - \\
\hline & & 2.7 & 63.6 & 15.5 & - & 4.8 & 14.5 & - & 43.3 & 5.2 & 16.6 & - & - & - & - \\
\hline 6 & $34.5-44.0$ & 36.0 & 41.6 & 7.8 & 62.7 & 2.7 & 8.1 & - & 11.0 & 2.1 & 1.8 & 3.7 & - & - & - \\
\hline 9 & $63.0-72.5$ & 65.8 & 36.5 & 8.0 & 60.8 & 2.6 & 8.4 & - & 11.8 & 2.0 & 2.2 & 4.3 & - & - & - \\
\hline 14 & $110.5-120.0$ & 113.0 & 40.5 & 10.5 & 45.5 & 3.5 & 10.8 & - & 19.1 & 3.3 & 5.5 & 1.9 & - & - & - \\
\hline 15 & $120.0-129.5$ & 122.8 & 45.4 & 13.7 & 29.6 & 6.3 & 15.4 & - & 22.6 & 4.2 & 5.9 & 1.0 & - & 1.4 & - \\
\hline \multirow[t]{2}{*}{19} & $158.0-167.5$ & 160.4 & 45.5 & 14.2 & 32.4 & 5.0 & 14.1 & - & 23.3 & 4.4 & 5.7 & 0.9 & - & - & - \\
\hline & & 163.3 & 43.1 & 14.4 & 27.4 & 6.2 & 16.3 & - & 20.6 & 4.5 & 9.3 & 0.7 & - & 0.7 & - \\
\hline 22 & $186.5-196.0$ & 188.0 & 55.7 & 20.1 & - & 10.7 & 33.6 & - & 14.9 & 4.8 & 14.3 & - & - & 1.5 & - \\
\hline 24 & $205.5-215.0$ & 207.1 & 49.5 & 21.1 & - & 6.7 & 14.4 & - & 38.7 & 7.2 & 11.2 & - & - & 0.7 & - \\
\hline 28 & $243.5-253.0$ & 248.2 & 52.2 & 23.6 & - & 6.3 & 18.5 & - & 34.4 & 6.8 & 10.5 & - & - & - & - \\
\hline 31 & $272.0-281.5$ & 275.1 & 49.0 & 23.0 & - & 7.7 & 16.1 & - & 35.0 & 6.5 & 10.2 & - & - & - & 1.4 \\
\hline 33 & $291.0-300.5$ & 294.0 & 45.9 & 24.2 & - & 5.8 & 14.4 & - & 38.2 & 7.2 & 10.2 & - & - & - & - \\
\hline 35 & $310.0-319.5$ & 315.1 & 33.8 & 36.7 & - & 11.2 & 19.2 & - & 21.4 & 3.7 & 7.8 & - & - & - & - \\
\hline 37 & $329.0-338.5$ & 332.5 & 33.5 & 33.3 & - & 8.6 & 17.6 & $\overline{-}$ & 28.1 & 5.3 & 7.1 & - & - & - & - \\
\hline 40 & $357.5-367.0$ & 363.6 & 49.7 & 20.0 & - & 7.3 & 11.0 & 2.2 & 39.8 & 6.9 & 12.8 & - & - & - & - \\
\hline 41 & $367.0-370.0$ & 369.7 & 49.1 & 19.5 & - & 5.4 & 8.2 & 3.4 & 43.1 & 7.3 & 13.0 & - & $\overline{-}$ & - & - \\
\hline \multirow[t]{2}{*}{43} & $376.5-386.0$ & 378.7 & 45.1 & 28.1 & - & 3.0 & 2.9 & 26.2 & 25.5 & - & 7.5 & - & 5.1 & 1.7 & - \\
\hline & & 383.7 & 43.0 & 24.4 & - & 4.9 & 2.8 & 20.3 & 36.2 & - & 7.9 & - & 2.7 & 0.8 & - \\
\hline 44 & $386.0-395.5$ & 387.3 & 12.9 & 3.5 & - & - & - & 36.3 & 15.1 & - & 44.0 & - & - & 1.1 & - \\
\hline
\end{tabular}

${ }^{\mathrm{a}} \mathrm{Cado}=$ calcium dolomite .

$\mathrm{b}_{\mathrm{U}-2}$ peak at $12.1 \AA$. 
TABLE 22

Results of X-Ray Diffraction Analysis From Site 271

\begin{tabular}{|c|c|c|c|c|c|c|c|c|c|c|c|}
\hline Core & $\begin{array}{c}\text { Cored } \\
\text { Interval } \\
\text { Below Sea } \\
\text { Floor (m) }\end{array}$ & $\begin{array}{l}\text { Sample Depth } \\
\text { Below Sea } \\
\text { Floor (m) }\end{array}$ & 嵌 & 荡 & $\begin{array}{l}\dot{0} \\
\frac{1}{\dot{1}}\end{array}$ & $\frac{m}{\pi}$ & 苋 & ह่ & 崖 & 范 & 总 \\
\hline \multicolumn{12}{|c|}{ Bulk Samples } \\
\hline 5 & $68.5-78.0$ & 69.6 & 34.1 & 33.0 & 18.1 & 22.5 & 23.0 & 2.3 & & & 1.1 \\
\hline 24 & $255.5-265.0$ & 258.3 & 39.2 & 23.8 & 10.5 & 21.8 & 31.1 & 2.2 & & & 10.5 \\
\hline \multicolumn{12}{|c|}{$2-20 \mu \mathrm{m}$ Fractions } \\
\hline 5 & $68.5-78.0$ & 69.6 & 24.3 & 38.5 & 13.1 & 25.1 & 19.1 & 2.6 & - & & 1.7 \\
\hline 24 & $255.5-265.0$ & 258.3 & 36.2 & 40.2 & 10.0 & 28.9 & 13.5 & 2.6 & 1.7 & & 3.1 \\
\hline \multicolumn{12}{|c|}{$<2 \mu \mathrm{m}$ Fractions } \\
\hline 5 & 68.5-78.0 & 69.6 & 76.9 & 8.3 & 3.5 & 7.5 & 25.8 & 54.9 & & $\overline{17}$ & \\
\hline 24 & $255.5-265.0$ & 258.3 & 80.9 & 17.4 & 6.9 & 20.5 & 27.7 & 9.8 & & 17.7 & \\
\hline
\end{tabular}

TABLE 23

Results of X-Ray Diffraction Analysis From Site $\mathbf{2 7 2}$

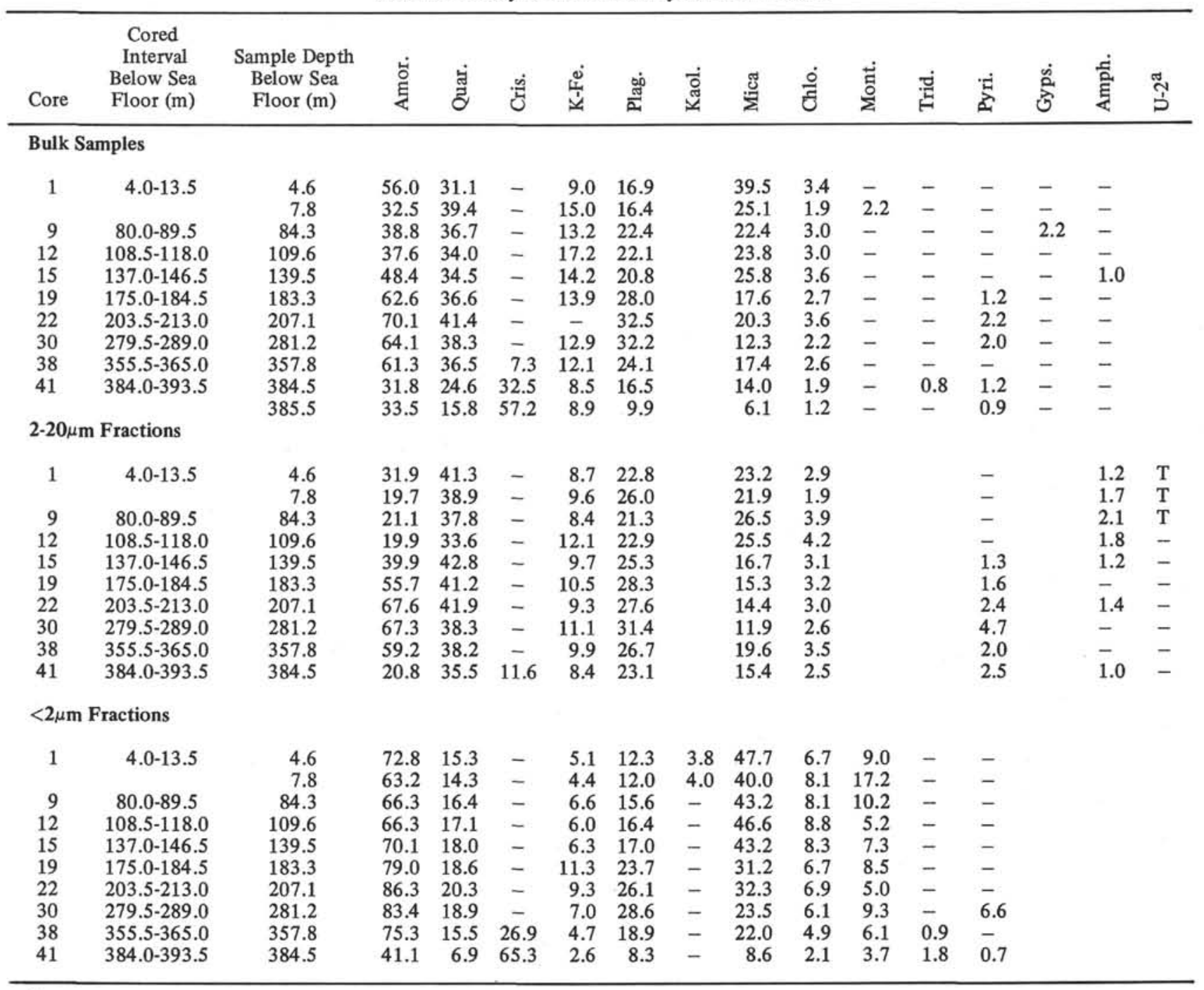

${ }^{\mathrm{a}} \mathrm{U}-2$ peak at $12.1 \AA$. 
TABLE 24

Results of X-Ray Diffraction Analysis From Hole 273

\begin{tabular}{|c|c|c|c|c|c|c|c|c|c|c|c|c|c|}
\hline Core & $\begin{array}{c}\text { Cored } \\
\text { Interval } \\
\text { Below Sea } \\
\text { Floor (m) }\end{array}$ & $\begin{array}{l}\text { Sample Depth } \\
\text { Below Sea } \\
\text { Floor (m) }\end{array}$ & 安 & 랠ㄹ & 这 & $\stackrel{\oplus 0}{\frac{0}{\alpha 2}}$ & 迅 & 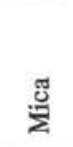 & ह่ & $\stackrel{+}{\dot{\Xi}}$ & 岕 & 䓂 & 芯 \\
\hline \multicolumn{14}{|c|}{ Bulk Samples } \\
\hline 2 & $4.5-14.0$ & 9.9 & 51.6 & 38.2 & 16.0 & 20.6 & & 23.2 & 2.0 & - & - & & - \\
\hline 4 & $23.5-33.0$ & 26.1 & 49.9 & 39.7 & 11.9 & 21.8 & & 20.1 & 1.9 & 1.1 & - & & 3.5 \\
\hline 6 & $42.5-52.0$ & 44.5 & 56.8 & 41.1 & 10.9 & 21.6 & & 19.1 & 2.5 & 1.3 & 1.1 & & 2.4 \\
\hline \multicolumn{14}{|c|}{$2-20 \mu \mathrm{m}$ Fractions } \\
\hline 4 & $23.5-33.0$ & 26.1 & 40.8 & 44.9 & 10.8 & 25.9 & & 13.6 & 2.4 & & 0.9 & 1.5 & \\
\hline 6 & $42.5-52.0$ & 44.5 & 39.0 & 42.9 & 12.6 & 24.3 & & 13.5 & 3.1 & & 2.2 & 1.4 & \\
\hline \multicolumn{14}{|c|}{$<2 \mu \mathrm{m}$ Fractions } \\
\hline 2 & $4.5-14.0$ & 9.9 & 77.0 & 22.6 & 6.7 & 19.3 & 1.1 & 31.6 & 5.5 & 13.3 & - & & \\
\hline 4 & $23.5-33.0$ & 26.1 & 79.6 & 22.3 & 7.5 & 17.9 & - & 30.7 & 6.0 & 15.5 & - & & \\
\hline 6 & $42.5-52.0$ & 44.5 & 75.7 & 22.0 & 8.0 & 21.0 & - & 28.6 & 6.2 & 10.6 & 3.5 & & \\
\hline
\end{tabular}

TABLE 25

Results of X-Ray Diffraction Analysis From Hole 273A

\begin{tabular}{|c|c|c|c|c|c|c|c|c|c|c|c|c|c|c|c|c|}
\hline Core & $\begin{array}{c}\text { Cored } \\
\text { Interval } \\
\text { Below Sea } \\
\text { Floor }(\mathrm{m})\end{array}$ & $\begin{array}{l}\text { Sample Depth } \\
\text { Below Sea } \\
\text { Floor (m) }\end{array}$ & 宾 & సुँ & ํํㅁ & है & 檪 & 离 & 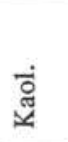 & ‡ூّ & $\frac{\dot{0}}{\text { ही }}$ & 茫 & 忘 & 官 & 竞 & $\stackrel{\widetilde{s}}{\rho}$ \\
\hline \multicolumn{17}{|c|}{ Bulk Samples } \\
\hline 6 & $137.5-147.0$ & 139.9 & 72.8 & - & - & 32.8 & 11.5 & 23.9 & - & 25.1 & 2.6 & - & 2.8 & 1.4 & - & \\
\hline 7 & $147.0-156.5$ & 149.0 & 53.7 & 4.4 & 30.3 & 28.2 & 10.5 & 18.8 & - & 6.9 & 0.9 & - & - & - & - & \\
\hline & & 149.5 & 55.1 & - & - & 40.4 & 14.0 & 26.7 & - & 14.6 & 1.6 & - & 1.7 & - & 1.0 & \\
\hline 9 & $166.0-175.5$ & 166.9 & 53.7 & - & - & 38.1 & 18.6 & 25.6 & 1.0 & 13.9 & 1.6 & 1.2 & - & - & - & \\
\hline 13 & $194.5-204.0$ & 198.8 & 53.1 & - & - & 38.5 & 14.2 & 24.4 & - & 15.2 & 2.2 & 3.6 & - & 1.9 & - & \\
\hline 17 & $232.5-242.0$ & 234.8 & 57.7 & - & - & 37.8 & 17.7 & 22.3 & - & 18.5 & 2.7 & 1.0 & - & - & - & \\
\hline 22 & $272.5-280.0$ & 273.3 & 30.3 & - & - & 31.5 & 25.0 & 22.5 & - & 19.6 & 1.4 & - & - & - & - & \\
\hline 25 & $299.0-308.5$ & 301.6 & 51.7 & 1.6 & 0.8 & 40.2 & 10.2 & 23.9 & - & 20.2 & 1.9 & - & - & - & 1.1 & \\
\hline \multicolumn{17}{|c|}{$2-20 \mu \mathrm{m}$ Fractions } \\
\hline 6 & $137.5-147.0$ & 139.9 & 65.3 & & - & 37.3 & 17.0 & 22.5 & & 12.7 & 2.4 & & 5.9 & & 2.1 & - \\
\hline 7 & $147.0-156.5$ & 149.0 & 43.1 & & 3.5 & 42.7 & 14.3 & 26.8 & & 7.1 & 1.6 & & 2.9 & & 1.2 & - \\
\hline & & 149.5 & 40.2 & & - & 38.4 & 12.3 & 26.0 & & 14.0 & 2.2 & & 5.4 & & 1.8 & - \\
\hline 9 & $166.0-175.5$ & 166.9 & 45.7 & & - & 36.0 & 19.5 & 25.3 & & 14.0 & 2.7 & & 1.2 & & 1.4 & - \\
\hline 13 & $194.5-204.0$ & 198.8 & 45.9 & & - & 39.0 & 12.6 & 27.4 & & 15.8 & 2.1 & & 1.4 & & 1.7 & $\mathbf{P}$ \\
\hline 17 & $232.5-242.0$ & 234.8 & 56.2 & & - & 44.0 & 11.3 & 25.8 & & 12.6 & 1.8 & & 3.2 & & 1.3 & $\mathbf{P}$ \\
\hline 22 & $272.5-280.0$ & 273.3 & 22.2 & & - & 34.4 & 13.2 & 27.1 & & 18.6 & 2.5 & & 1.8 & & 2.2 & $\mathbf{P}$ \\
\hline 25 & $299.0-308.5$ & 301.6 & 42.0 & & - & 38.3 & 12.2 & 24.9 & & 18.9 & 3.0 & & 0.9 & & 1.8 & $\mathbf{P}$ \\
\hline \multicolumn{17}{|c|}{$<2 \mu \mathrm{m}$ Fractions } \\
\hline 6 & $137.5-147.0$ & 139.9 & 85.1 & & - & 24.8 & 9.3 & 22.4 & - & 24.5 & 5.6 & 9.8 & 3.6 & & & \\
\hline 7 & $147.0-156.5$ & 149.0 & 83.3 & & 3.0 & 27.5 & 8.1 & 25.8 & - & 18.0 & 5.5 & 9.7 & 2.4 & & & \\
\hline & & 149.5 & 76.3 & & - & 23.0 & 8.9 & 23.4 & - & 21.2 & 5.3 & 16.8 & 1.3 & & & \\
\hline 9 & $166.0-175.5$ & 166.9 & 76.9 & & - & 22.8 & 9.6 & 22.0 & 3.1 & 24.2 & 5.4 & 12.9 & - & & & \\
\hline 13 & $194.5-204.0$ & 198.8 & 75.4 & & - & 21.8 & 8.7 & 21.1 & 2.3 & 26.8 & 5.8 & 13.5 & - & & & \\
\hline 17 & $232.5-242.0$ & 234.8 & 78.0 & & - & 20.6 & 10.7 & 19.3 & - & 30.3 & 6.8 & 10.9 & 1.4 & & & \\
\hline 22 & $272.5-280.0$ & 273.3 & 73.5 & & - & 21.3 & 6.4 & 23.0 & - & 34.0 & 6.9 & 8.3 & - & & & \\
\hline 25 & $299.0-308.5$ & 301.6 & 66.8 & & - & 16.3 & 6.9 & 21.7 & - & 41.0 & 5.5 & 8.6 & - & & & \\
\hline
\end{tabular}

${ }^{\mathrm{a}} \mathrm{U}-3$ peaks at $22.2 \AA$ and $11.25 \AA$. 
TABLE 26

Results of X-Ray Diffraction Analysis From Site 274

\begin{tabular}{|c|c|c|c|c|c|c|c|c|c|c|c|c|c|c|c|c|c|c|c|c|}
\hline Core & $\begin{array}{c}\text { Cored } \\
\text { Interval } \\
\text { Below Sea } \\
\text { Floor }(\mathrm{m})\end{array}$ & $\begin{array}{l}\text { Sample Depth } \\
\text { Below Sea } \\
\text { Floor }(\mathrm{m})\end{array}$ & 衣 & $\stackrel{\grave{0}}{\circ}$ & मूँ & 咅 & है & $\frac{\dot{j}}{\dot{x}}$ & $\frac{30}{2}$ & $\begin{array}{l}\dot{s} \\
\check{g}\end{array}$ & $\stackrel{\mathrm{J}}{\frac{\mathrm{N}}{2}}$ & है & $\stackrel{\overrightarrow{\tilde{c}}}{\stackrel{c}{\Sigma}}$ & 总 & E & 定 & $\sum_{0}^{2}$ & 肎 & $\stackrel{\tilde{y}}{3}$ & $\stackrel{5}{3}$ \\
\hline \multicolumn{21}{|c|}{ Bulk Samples } \\
\hline 1 & $0.0-9.5$ & 5.7 & 54.3 & - & - & 28.1 & - & 15.3 & 18.1 & - & 29.8 & 2.5 & 4.8 & - & - & - & - & 1.4 & & \\
\hline 2 & $9.5-19.0$ & 13.2 & 53.9 & - & - & 30.1 & - & 11.3 & 19.5 & 1.6 & 30.8 & 3.0 & 3.8 & - & - & - & - & - & & \\
\hline 5 & $38.0-47.5$ & 42.3 & 67.0 & - & - & 28.3 & - & 11.2 & 21.2 & - & 35.2 & 2.3 & 1.9 & - & - & - & - & - & & \\
\hline 6 & $47.5-57.0$ & 50.3 & 60.6 & - & - & 28.6 & - & 12.6 & 21.9 & - & 33.1 & 2.0 & 1.8 & - & - & - & - & - & & \\
\hline 10 & $85.5-95.0$ & 89.4 & 69.1 & - & - & 30.7 & - & 10.5 & 22.1 & - & 30.3 & 1.5 & 4.8 & - & - & - & - & - & & \\
\hline 13 & $114.0-123.5$ & 116.6 & 56.4 & - & - & 30.7 & - & 11.6 & 21.6 & - & 30.9 & 1.2 & 2.9 & - & - & - & - & 1.1 & & \\
\hline & & 122.6 & 58.5 & - & - & 32.7 & - & 11.4 & 21.9 & - & 28.3 & 0.7 & 3.6 & - & - & - & - & 1.2 & & \\
\hline 14 & $123.5-133.0$ & 125.9 & 60.7 & - & - & 32.0 & - & 9.2 & 22.9 & - & 30.2 & 3.5 & 1.1 & - & - & - & - & 1.0 & & \\
\hline 17 & $152.0-161.5$ & 154.3 & 57.1 & - & - & 29.4 & - & 8.4 & 21.5 & - & 35.2 & 4.2 & $=$ & - & - & - & - & 1.3 & & \\
\hline 20 & $180.5-190.0$ & 184.4 & 58.0 & - & - & 26.1 & - & 7.7 & 18.4 & - & 29.0 & 2.0 & 16.9 & - & - & - & - & - & & \\
\hline 21 & $190.0-199.5$ & 198.2 & 77.5 & - & - & 35.7 & - & 9.9 & 19.5 & - & 27.2 & 3.7 & 2.0 & - & - & 2.0 & - & - & & \\
\hline 23 & $209.0-218.5$ & 211.9 & 81.0 & - & - & 36.6 & - & 7.8 & 21.7 & - & 27.7 & 3.8 & - & - & - & 2.5 & - & - & & \\
\hline 26 & $237.5 \cdot 247.0$ & 241.4 & 80.4 & - & 1.6 & 34.9 & - & 11.0 & 20.4 & - & 26.7 & 3.3 & - & - & - & 2.1 & - & - & & \\
\hline 29 & $266.0-275.5$ & 267.8 & 83.7 & - & 1.5 & 33.5 & - & 8.9 & 19.8 & - & 27.7 & 3.0 & 2.3 & - & - & 3.3 & - & - & & \\
\hline 32 & $294.5-304.0$ & 300.4 & 78.0 & - & - & 31.4 & - & 9.3 & 18.6 & - & 29.1 & 2.5 & 7.3 & - & - & 1.8 & - & - & & \\
\hline 39 & $361.0-370.5$ & 363.6 & 44.0 & 4.1 & 3.3 & 26.2 & 12.0 & 8.5 & 11.6 & - & 21.9 & 2.2 & 6.4 & 0.8 & 0.9 & 2.1 & - & - & & \\
\hline 42 & $389.5-399.0$ & 392.4 & 29.6 & - & - & 12.7 & 39.9 & 3.7 & 6.0 & - & 15.9 & 1.0 & 13.2 & 2.1 & 2.0 & - & 3.4 & - & & \\
\hline \multicolumn{21}{|c|}{$2.20 \mu \mathrm{m}$ Fractions } \\
\hline 1 & $0.0-9.5$ & 5.7 & 33.6 & & - & 37.7 & - & 10.2 & 24.3 & & 23.1 & 2.7 & & - & - & - & & 2.0 & - & - \\
\hline 2 & $9.5-19.0$ & 13.2 & 27.9 & & - & 40.2 & - & 9.9 & 24.9 & & 20.7 & 2.6 & & - & - & - & & 1.7 & - & $\mathrm{P}$ \\
\hline 5 & $38.0-47.5$ & 42.3 & 55.9 & & - & 35.5 & - & 10.8 & 24.9 & & 24.3 & 2.9 & & - & - & - & & 1.5 & - & $\mathbf{P}$ \\
\hline 6 & $47.5-57.0$ & 50.3 & 42.9 & & - & 41.6 & - & 10.4 & 25.9 & & 18.4 & 2.1 & & - & - & - & & 1.5 & - & - \\
\hline 10 & $85.5-95.0$ & 89.4 & 49.0 & & - & 35.3 & - & 10.6 & 25.3 & & 26.1 & 1.0 & & - & - & - & & 1.7 & - & - \\
\hline 13 & $114.0-123.5$ & 116.6 & 30.4 & & - & 40.2 & - & 11.8 & 24.7 & & 19.7 & 1.3 & & - & - & - & & 2.3 & - & - \\
\hline & & 122.6 & 40.9 & & - & 40.0 & - & 10.9 & 27.4 & & 19.0 & 1.3 & & - & - & - & & 1.4 & - & - \\
\hline 14 & $123.5-133.0$ & 125.9 & 35.4 & & - & 39.8 & - & 11.8 & 26.1 & & 16.1 & 3.1 & & - & - & 0.9 & & 2.1 & - & P \\
\hline 17 & $152.0-161.5$ & 154.3 & 32.5 & & - & 34.2 & - & 12.2 & 25.9 & & 21.4 & 4.9 & & - & - & - & & 1.4 & $T$ & - \\
\hline 20 & $180.5-190.0$ & 184.4 & 56.8 & & - & 44.6 & - & 9.3 & 26.8 & & 17.5 & 1.7 & & - & - & - & & - & - & $T$ \\
\hline 21 & $190.0-199.5$ & 198.2 & 72.4 & & - & 46.6 & - & 10.5 & 22.8 & & 12.9 & 3.2 & & - & - & 4.0 & & - & - & - \\
\hline 23 & $209.0-218.5$ & 211.9 & 76.5 & & - & 49.2 & - & 9.7 & 22.6 & & 11.2 & 3.8 & & - & - & 3.5 & & - & - & P \\
\hline 26 & $237.5-247.0$ & 241.4 & 78.3 & & - & 47.1 & - & 11.7 & 22.9 & & 10.9 & 3.6 & & - & - & 3.8 & & - & - & - \\
\hline 29 & $266.0-275.5$ & 267.8 & 82.2 & & - & 43.8 & - & 12.5 & 25.7 & & 11.0 & 2.4 & & - & - & 4.6 & & - & - & $T$ \\
\hline 32 & $294.5-304.0$ & 300.4 & 71.7 & & - & 49.3 & - & 10.7 & 22.8 & & 12.0 & 1.7 & & - & - & 3.4 & & - & - & P \\
\hline 39 & $361.0-370.5$ & 363.6 & 21.6 & & 1.8 & 37.6 & 4.9 & 10.9 & 19.4 & & 16.5 & 2.6 & & 0.6 & 0.9 & 4.7 & & - & - & $\mathbf{P}$ \\
\hline 42 & $389.5-399.0$ & 392.4 & 22.1 & & - & 20.3 & 37.1 & 7.0 & 10.4 & & 15.7 & 2.0 & & 3.2 & 2.5 & 1.9 & & - & - & - \\
\hline \multicolumn{21}{|c|}{$<2 \mu \mathrm{m}$ Fractions } \\
\hline 1 & $0.0-9.5$ & 5.7 & 64.7 & & & 12.5 & - & 6.1 & 11.9 & 3.1 & 35.9 & 6.1 & 24.3 & - & - & - & & & & \\
\hline 2 & $9.5-19.0$ & 13.2 & 66.5 & & & 12.7 & - & 6.5 & 12.0 & 4.5 & 34.8 & 6.2 & 23.2 & - & - & - & & & & \\
\hline 5 & $38.0-47.5$ & 42.3 & 79.8 & & & 16.2 & - & 4.7 & 15.2 & 5.9 & 32.2 & 1.9 & 23.9 & - & - & - & & & & \\
\hline 6 & $47.5-57.0$ & 50.3 & 73.9 & & & 15.7 & - & 7.6 & 15.5 & 3.8 & 30.3 & 2.1 & 25.0 & - & - & - & & & & \\
\hline 10 & $85.5-95.0$ & 89.4 & 78.9 & & & 15.2 & - & 8.2 & 14.4 & 5.1 & 29.7 & - & 27.4 & - & - & - & & & & \\
\hline \multirow[t]{2}{*}{13} & $114.0-123.5$ & 116.6 & 72.8 & & & 19.2 & - & 7.5 & 18.3 & 3.1 & 28.2 & 1.5 & 22.3 & - & - & - & & & & \\
\hline & & 122.6 & 70.7 & & & 17.5 & - & 10.9 & 16.7 & 3.2 & 24.3 & 1.3 & 26.2 & - & - & - & & & & \\
\hline 14 & $123.5-133.0$ & 125.9 & 74.9 & & & 20.3 & - & 9.1 & 21.4 & - & 31.0 & 6.0 & 12.2 & - & - & - & & & & \\
\hline 17 & $152.0-161.5$ & 154.3 & 72.8 & & & 21.4 & - & 7.8 & 21.1 & - & 37.6 & 7.2 & 4.9 & - & - & - & & & & \\
\hline 20 & $180.5-190.0$ & 184.4 & 52.7 & & & 15.0 & - & 5.7 & 9.6 & 1.0 & 33.4 & 1.8 & 33.3 & - & - & - & & & & \\
\hline 21 & $190.0-199.5$ & 198.2 & 80.0 & & & 31.2 & - & 7.8 & 20.8 & $\ldots$ & 25.3 & 4.7 & 9.1 & - & - & 1.2 & & & & \\
\hline 23 & $209.0-218.5$ & 211.9 & 81.6 & & & 27.9 & - & 7.5 & 18.0 & - & 26.3 & 7.2 & 11.5 & - & - & 1.6 & & & & \\
\hline 26 & $237.5-247.0$ & 241.4 & 80.9 & & & 26.6 & - & 6.6 & 17.3 & - & 28.3 & 4.6 & 15.2 & - & - & 1.4 & & & & \\
\hline 29 & $266.0-275.5$ & 267.8 & 79.6 & & & 23.5 & - & 8.3 & 18.1 & - & 30.3 & 4.8 & 13.2 & - & - & 1.8 & & & & \\
\hline 32 & $294.5-304.0$ & 300.4 & 76.2 & & & 22.1 & - & 5.1 & 15.6 & - & 28.0 & 2.9 & 24.9 & - & - & 1.5 & & & & \\
\hline 39 & $361.0-370.5$ & 363.6 & 47.9 & & & 21.6 & 23.8 & 5.1 & 10.5 & - & 18.1 & 2.4 & 14.8 & 0.7 & 1.3 & 1.8 & & & & \\
\hline 42 & $389.5-399.0$ & 392.4 & 39.5 & & & 7.0 & 60.7 & 2.2 & 5.2 & - & 7.6 & 0.6 & 11.2 & 4.8 & - & 0.7 & & & & \\
\hline
\end{tabular}

${ }^{\mathrm{a} U} \mathrm{U}-2$ peak at $12.1 \AA$.

${ }^{b} \mathrm{U}-3$ peaks at $22.2 \AA$ and $11.25 \AA$. 